\title{
Striking Difference between Succinimidomethyl and Phthalimidomethyl Radicals in Conjugate Addition to Alkylidenemalonate Initiated by Dimethylzinc
}

Ken-ichi Yamada*, Yusuke Matsumoto, Shintaro Fujii, Takehito Konishi, Yosuke Yamaoka, and

\author{
Kiyosei Takasu
}

Graduate School of Pharmaceutical Sciences, Kyoto University Yoshida, Sakyo-ku, Kyoto 606-8501, Japan

E-mail: yamak@pharm.kyoto-u.ac.jp

Table of Contents

Details of DFT Calclulations S2

NMR Charts S8

ORTEP Drawing of $\mathbf{8}$ S58

Reference S59 


\section{Supporting Information}

DFT Calculations. All the calculations were performed using Gaussian 09W program. ${ }^{1}$ The geometry optimization and the frequency analyses were conducted at B3LYP/6-31+G(d) level of theory, while the energies and the natural population were calculated at B3LYP/6-311++G(3df,3pd) level of theory.

\section{$N$-Methylsuccinimide:}

Zero-point vibrational energy

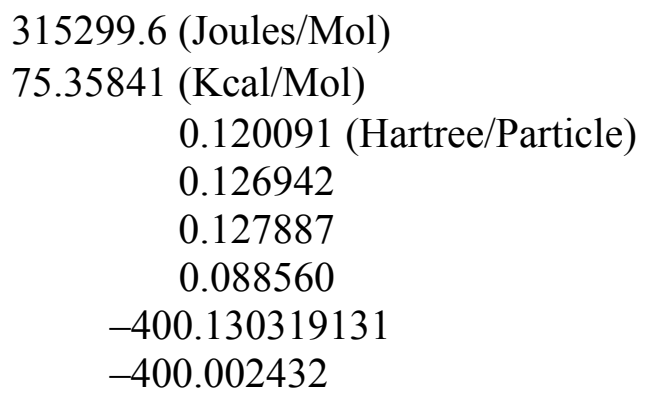

Sum of electronic and thermal Enthalpies =

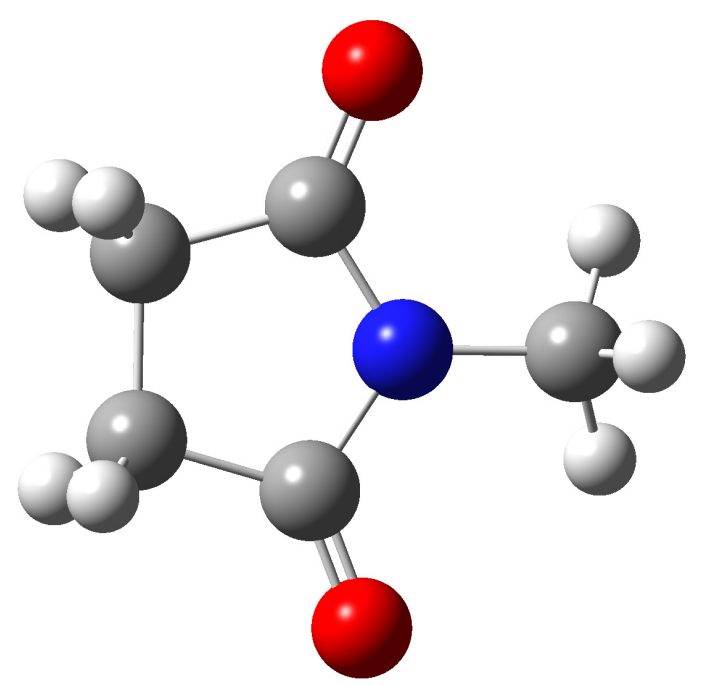

\begin{tabular}{crrr}
\hline $\begin{array}{c}\text { Atomic } \\
\text { Type }\end{array}$ & $\mathrm{X}$ & $\mathrm{Y}$ & $\mathrm{Z}$ \\
\hline $\mathrm{H}$ & 1.022959 & 2.467186 & 0.000000 \\
$\mathrm{C}$ & -0.002528 & 2.082192 & 0.000000 \\
$\mathrm{H}$ & -0.514494 & 2.430054 & 0.898946 \\
$\mathrm{H}$ & -0.514494 & 2.430054 & -0.898946 \\
$\mathrm{~N}$ & -0.003998 & 0.625570 & 0.000000 \\
$\mathrm{C}$ & 0.002166 & -0.138760 & -1.166916 \\
$\mathrm{C}$ & 0.002166 & -0.138760 & 1.166916 \\
$\mathrm{C}$ & -0.000188 & -1.610831 & -0.768795 \\
$\mathrm{H}$ & 0.879019 & -2.094689 & -1.206303 \\
$\mathrm{H}$ & -0.881178 & -2.091809 & -1.205934 \\
$\mathrm{C}$ & -0.000188 & -1.610831 & 0.768795 \\
$\mathrm{H}$ & 0.879019 & -2.094689 & 1.206303 \\
$\mathrm{H}$ & -0.881178 & -2.091809 & 1.205934 \\
$\mathrm{O}$ & 0.004055 & 0.321924 & -2.292079 \\
$\mathrm{O}$ & 0.004055 & 0.321924 & 2.292079 \\
\hline
\end{tabular}




\section{Supporting Information}

Succinimidomethyl:

Zero-point vibrational energy

Zero-point correction $=$

Thermal correction to Energy $=$

Thermal correction to Enthalpy =

Thermal correction to Gibbs Free Energy =

Energies (UB3LYP) $=$

Sum of electronic and thermal Enthalpies =

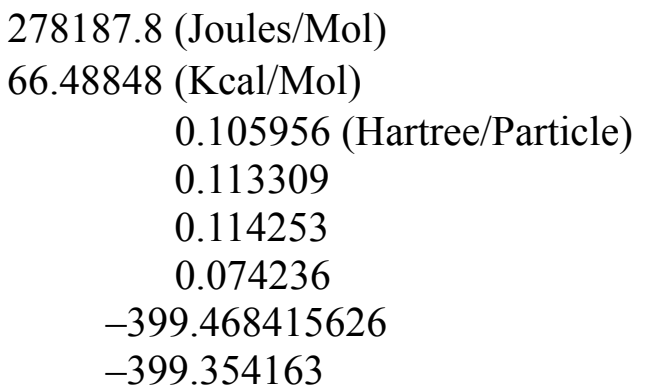

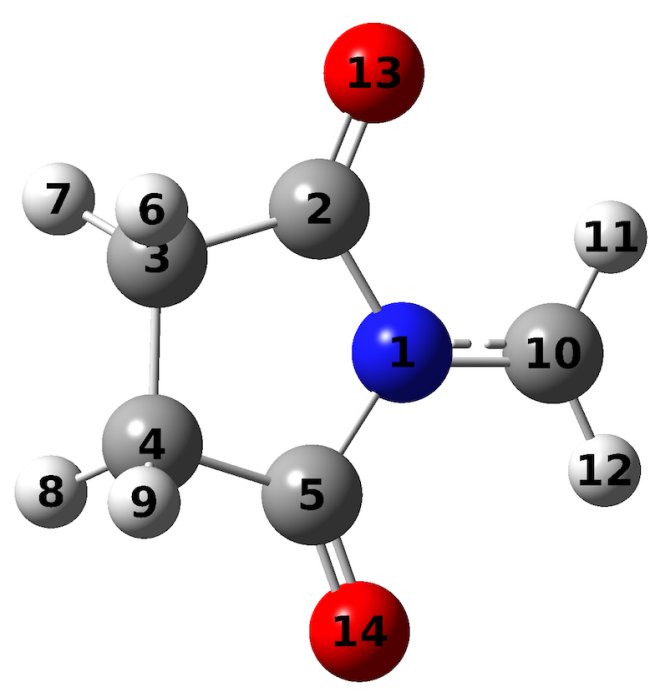

\begin{tabular}{ccrrr}
\hline $\begin{array}{c}\text { Atomic } \\
\text { Type }\end{array}$ & No & \multicolumn{3}{c}{ Coordinates (Angstroms) } \\
\hline $\mathrm{N}$ & 1 & $\mathrm{X}$ & $\mathrm{Y}$ & $\mathrm{Z}$ \\
$\mathrm{C}$ & 2 & 0.000000 & 0.000000 & 0.687190 \\
$\mathrm{C}$ & 3 & 0.000000 & 1.174819 & -0.098915 \\
$\mathrm{C}$ & 4 & 0.000000 & 0.768683 & -1.563885 \\
$\mathrm{C}$ & 5 & 0.000000 & -0.768683 & -1.563885 \\
$\mathrm{H}$ & 6 & 0.000000 & -1.174819 & -0.098915 \\
$\mathrm{H}$ & 7 & -0.879963 & 1.203166 & -2.050344 \\
$\mathrm{H}$ & 8 & 0.879963 & 1.203166 & -2.050344 \\
$\mathrm{H}$ & 9 & 0.879963 & -1.203166 & -2.050344 \\
$\mathrm{C}$ & 10 & -0.879963 & -1.203166 & -2.050344 \\
$\mathrm{H}$ & 11 & 0.000000 & 0.000000 & 2.062265 \\
$\mathrm{H}$ & 12 & 0.000000 & 0.958509 & 2.559253 \\
$\mathrm{O}$ & 13 & 0.000000 & -0.958509 & 2.559253 \\
$\mathrm{O}$ & 14 & 0.000000 & 2.295868 & 0.365785 \\
\hline
\end{tabular}




\section{Supporting Information}

Summary of Natural Population Analysis of $\alpha$ Spin Orbitals

\begin{tabular}{ccrrrrr}
\hline Atomic & & & \multicolumn{4}{c}{ Natural Population } \\
\cline { 4 - 6 } Type & No & Natural Charge & Core & Valence & Rydberg & Total \\
\hline $\mathrm{N}$ & 1 & -0.25665 & 0.99958 & 2.74359 & 0.01347 & 3.75665 \\
$\mathrm{C}$ & 2 & 0.33331 & 0.99967 & 1.64677 & 0.02025 & 2.66669 \\
$\mathrm{C}$ & 3 & -0.23560 & 0.99960 & 2.22737 & 0.00862 & 3.23560 \\
$\mathrm{C}$ & 4 & -0.23560 & 0.99960 & 2.22737 & 0.00862 & 3.23560 \\
$\mathrm{C}$ & 5 & 0.33331 & 0.99967 & 1.64677 & 0.02025 & 2.66669 \\
$\mathrm{H}$ & 6 & 0.11469 & 0.00000 & 0.38397 & 0.00134 & 0.38531 \\
$\mathrm{H}$ & 7 & 0.11469 & 0.00000 & 0.38397 & 0.00134 & 0.38531 \\
$\mathrm{H}$ & 8 & 0.11469 & 0.00000 & 0.38397 & 0.00134 & 0.38531 \\
$\mathrm{H}$ & 9 & 0.11469 & 0.00000 & 0.38397 & 0.00134 & 0.38531 \\
$\mathrm{C}$ & 10 & -0.49242 & 0.99969 & 2.47645 & 0.01628 & 3.49242 \\
$\mathrm{H}$ & 11 & 0.11095 & 0.00000 & 0.38690 & 0.00216 & 0.38905 \\
$\mathrm{H}$ & 12 & 0.11095 & 0.00000 & 0.38690 & 0.00216 & 0.38905 \\
$\mathrm{O}$ & 13 & -0.31351 & 0.99987 & 3.29791 & 0.01573 & 4.31351 \\
$\mathrm{O}$ & 14 & -0.31351 & 0.99987 & 3.29791 & 0.01573 & 4.31351 \\
\hline Total & & -0.50000 & 7.99756 & 21.87383 & 0.12862 & 30.0000 \\
\hline
\end{tabular}

Summary of Natural Population Analysis of $\beta$ Spin Orbitals

\begin{tabular}{ccrrrrr}
\hline Atomic & & & \multicolumn{4}{c}{ Natural Population } \\
\cline { 4 - 6 } Type & No & Natural Charge & Core & Valence & Rydberg & Total \\
\hline $\mathrm{N}$ & 1 & -0.26779 & 0.99958 & 2.75770 & 0.01051 & 3.76779 \\
$\mathrm{C}$ & 2 & 0.37752 & 0.99968 & 1.60350 & 0.01931 & 2.62248 \\
$\mathrm{C}$ & 3 & -0.23941 & 0.99960 & 2.23183 & 0.00798 & 3.23941 \\
$\mathrm{C}$ & 4 & -0.23941 & 0.99960 & 2.23183 & 0.00798 & 3.23941 \\
$\mathrm{C}$ & 5 & 0.37752 & 0.99968 & 1.60350 & 0.01931 & 2.62248 \\
$\mathrm{H}$ & 6 & 0.11945 & 0.00000 & 0.37933 & 0.00122 & 0.38055 \\
$\mathrm{H}$ & 7 & 0.11945 & 0.00000 & 0.37933 & 0.00122 & 0.38055 \\
$\mathrm{H}$ & 8 & 0.11945 & 0.00000 & 0.37933 & 0.00122 & 0.38055 \\
$\mathrm{H}$ & 9 & 0.11945 & 0.00000 & 0.37933 & 0.00122 & 0.38055 \\
$\mathrm{C}$ & 10 & 0.36559 & 0.99969 & 1.62546 & 0.00926 & 2.63441 \\
$\mathrm{H}$ & 11 & 0.08799 & 0.00000 & 0.41088 & 0.00114 & 0.41201 \\
$\mathrm{H}$ & 12 & 0.08799 & 0.00000 & 0.41088 & 0.00114 & 0.41201 \\
$\mathrm{O}$ & 13 & -0.26389 & 0.99987 & 3.24947 & 0.01454 & 4.26389 \\
$\mathrm{O}$ & 14 & -0.26389 & 0.99987 & 3.24947 & 0.01454 & 4.26389 \\
\hline Total & & 0.50000 & 7.99757 & 20.89184 & 0.11060 & 29.00000 \\
\hline
\end{tabular}




\section{Supporting Information}

$N$-Methylphthalimide:

Zero-point vibrational energy

377912.8 (Joules/Mol)

90.32332 (Kcal/Mol)

Zero-point correction $=$

Thermal correction to Energy $=$

0.143939 (Hartree/Particle)

Thermal correction to Enthalpy =

0.152789

Thermal correction to Gibbs Free Energy =

0.153734

Energies $($ RB3LYP $)=$

0.109737

Sum of electronic and thermal Enthalpies $=$

$-552.445459$

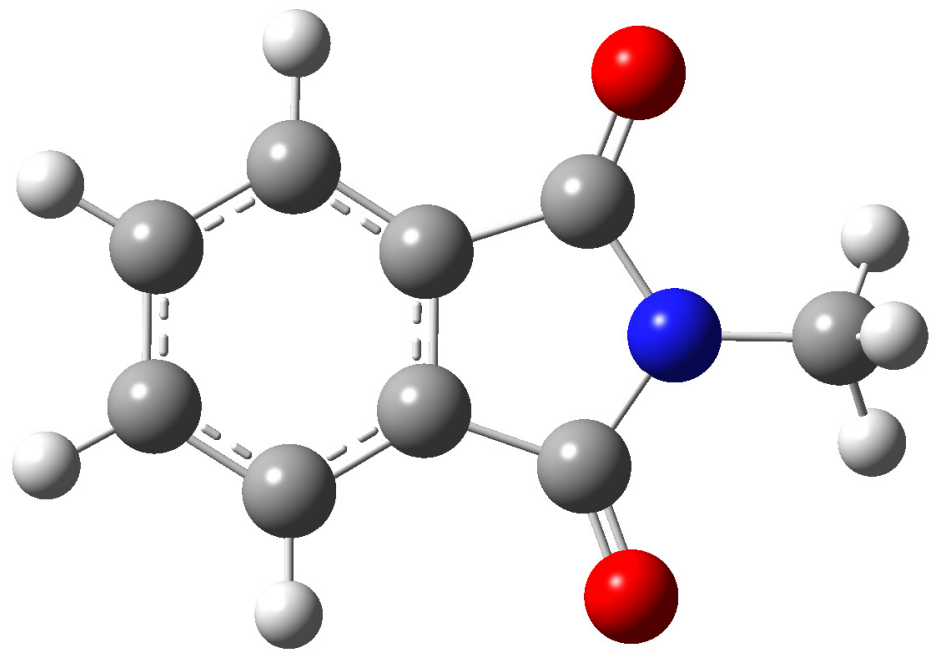

\begin{tabular}{crrr}
\hline $\begin{array}{c}\text { Atomic } \\
\text { Type }\end{array}$ & $\mathrm{X}$ & $\mathrm{Y}$ & $\mathrm{Z}$ \\
\hline $\mathrm{H}$ & -3.513035 & 1.032334 & 0.000000 \\
$\mathrm{C}$ & -3.130194 & 0.006025 & 0.000000 \\
$\mathrm{H}$ & -3.483065 & -0.506272 & 0.896944 \\
$\mathrm{H}$ & -3.483065 & -0.506272 & -0.896944 \\
$\mathrm{~N}$ & -1.676377 & -0.006303 & 0.000000 \\
$\mathrm{C}$ & -0.892526 & -0.001207 & -1.164377 \\
$\mathrm{C}$ & -0.892526 & -0.001207 & 1.164377 \\
$\mathrm{O}$ & -1.325979 & -0.001565 & -2.301403 \\
$\mathrm{O}$ & -1.325979 & -0.001565 & 2.301403 \\
$\mathrm{C}$ & 0.527382 & -0.000328 & 0.698783 \\
$\mathrm{C}$ & 2.912376 & 0.001842 & -0.700979 \\
$\mathrm{C}$ & 1.710438 & 0.000246 & 1.425031 \\
$\mathrm{C}$ & 0.527382 & -0.000328 & -0.698783 \\
$\mathrm{C}$ & 1.710438 & 0.000246 & -1.425031 \\
$\mathrm{C}$ & 2.912376 & 0.001842 & 0.700979 \\
$\mathrm{H}$ & 1.700131 & -0.000048 & 2.511034 \\
$\mathrm{H}$ & 1.700131 & -0.000048 & -2.511034 \\
$\mathrm{H}$ & 3.859165 & 0.003333 & 1.234310 \\
$\mathrm{H}$ & 3.859165 & 0.003333 & -1.234310 \\
\hline & & &
\end{tabular}




\section{Supporting Information}

Phthalimidomethyl:

Zero-point vibrational energy 340753.6 (Joules/Mol)

81.44207 (Kcal/Mol)

Zero-point correction $=$

Thermal correction to Energy $=$

0.129786 (Hartree/Particle)

Thermal correction to Enthalpy =

0.139123

Thermal correction to Gibbs Free Energy =

0.140067

Energies (UB3LYP) $=$

0.095495

Sum of electronic and thermal Enthalpies $=$

$-551.799211$

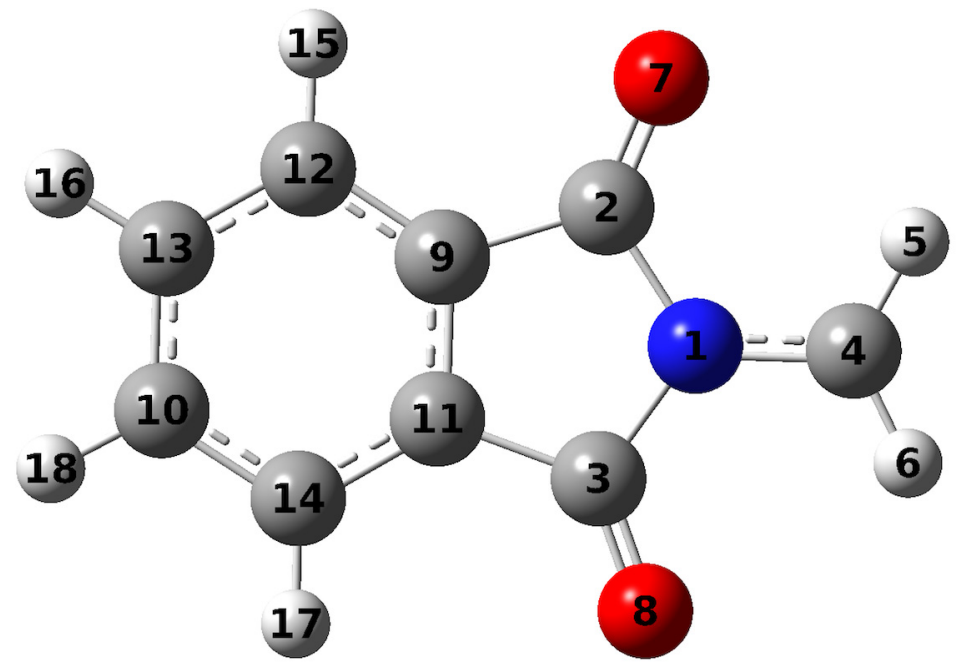

\begin{tabular}{ccrrr}
\hline $\begin{array}{c}\text { Atomic } \\
\text { Type }\end{array}$ & No & \multicolumn{3}{c}{ Coordinates (Angstroms) } \\
\hline $\mathrm{N}$ & 1 & $\mathrm{X}$ & $\mathrm{Y}$ & $\mathrm{Z}$ \\
$\mathrm{C}$ & 2 & 0.000000 & 0.000000 & 1.734061 \\
$\mathrm{C}$ & 3 & 0.000000 & 1.173694 & 0.929719 \\
$\mathrm{C}$ & 4 & 0.000000 & -1.173694 & 0.929719 \\
$\mathrm{H}$ & 5 & 0.000000 & 0.000000 & 3.104807 \\
$\mathrm{H}$ & 6 & 0.000000 & 0.957468 & 3.603886 \\
$\mathrm{O}$ & 7 & 0.000000 & -0.957468 & 3.603886 \\
$\mathrm{O}$ & 8 & 0.000000 & 2.306206 & 1.369247 \\
$\mathrm{C}$ & 9 & 0.000000 & -2.306206 & 1.369247 \\
$\mathrm{C}$ & 10 & 0.000000 & 0.699829 & -0.479850 \\
$\mathrm{C}$ & 11 & 0.000000 & -0.700296 & -2.866183 \\
$\mathrm{C}$ & 12 & 0.000000 & -0.699829 & -0.479850 \\
$\mathrm{C}$ & 13 & 0.000000 & 1.424943 & -1.663016 \\
$\mathrm{C}$ & 14 & 0.000000 & 0.700296 & -2.866183 \\
$\mathrm{H}$ & 15 & 0.000000 & -1.424943 & -1.663016 \\
$\mathrm{H}$ & 16 & 0.000000 & 2.510791 & -1.653054 \\
$\mathrm{H}$ & 17 & 0.000000 & 1.234269 & -3.812459 \\
$\mathrm{H}$ & 18 & 0.000000 & -2.510791 & -1.653054 \\
\hline
\end{tabular}




\section{Supporting Information}

Summary of Natural Population Analysis of $\alpha$ Spin Orbitals

\begin{tabular}{ccrcccr}
\hline Atomic & & & \multicolumn{4}{c}{ Natural Population } \\
\cline { 4 - 6 } Type & No & Natural Charge & Core & Valence & Rydberg & Total \\
\hline $\mathrm{N}$ & 1 & -0.25775 & 0.99961 & 2.74547 & 0.01267 & 3.75775 \\
$\mathrm{C}$ & 2 & 0.32688 & 0.99961 & 1.65329 & 0.02022 & 2.67312 \\
$\mathrm{C}$ & 3 & 0.32670 & 0.99961 & 1.65346 & 0.02023 & 2.67330 \\
$\mathrm{C}$ & 4 & -0.48458 & 0.99968 & 2.46856 & 0.01634 & 3.48458 \\
$\mathrm{H}$ & 5 & 0.11048 & 0.00000 & 0.38734 & 0.00218 & 0.38952 \\
$\mathrm{H}$ & 6 & 0.11049 & 0.00000 & 0.38734 & 0.00217 & 0.38951 \\
$\mathrm{O}$ & 7 & -0.29608 & 0.99987 & 3.28127 & 0.01494 & 4.29608 \\
$\mathrm{O}$ & 8 & -0.29602 & 0.99987 & 3.28121 & 0.01494 & 4.29602 \\
$\mathrm{C}$ & 9 & -0.05705 & 0.99941 & 2.04661 & 0.01103 & 3.05705 \\
$\mathrm{C}$ & 10 & -0.09961 & 0.99958 & 2.09097 & 0.00906 & 3.09961 \\
$\mathrm{C}$ & 11 & -0.05446 & 0.99941 & 2.04444 & 0.01061 & 3.05446 \\
$\mathrm{C}$ & 12 & -0.08822 & 0.99942 & 2.07789 & 0.01090 & 3.08822 \\
$\mathrm{C}$ & 13 & -0.10284 & 0.99958 & 2.09372 & 0.00953 & 3.10284 \\
$\mathrm{C}$ & 14 & -0.09374 & 0.99951 & 2.08468 & 0.00954 & 3.09374 \\
$\mathrm{H}$ & 15 & 0.11773 & 0.00000 & 0.38077 & 0.00150 & 0.38227 \\
$\mathrm{H}$ & 16 & 0.11009 & 0.00000 & 0.38895 & 0.00095 & 0.38991 \\
$\mathrm{H}$ & 17 & 0.11785 & 0.00000 & 0.38065 & 0.00150 & 0.38215 \\
$\mathrm{H}$ & 18 & 0.11016 & 0.00000 & 0.38885 & 0.00099 & 0.38984 \\
\hline Total & & -0.49996 & 11.99518 & 29.83547 & 0.16931 & 41.99996 \\
\hline & & & & &
\end{tabular}

Summary of Natural Population Analysis of $\beta$ Spin Orbitals

\begin{tabular}{crrrrrr}
\hline Atomic & & & \multicolumn{4}{c}{ Natural Population } \\
\cline { 5 - 7 } Type & No & Natural Charge & Core & Valence & Rydberg & Total \\
\hline $\mathrm{N}$ & 1 & -0.25437 & 0.99961 & 2.74498 & 0.00978 & 3.75437 \\
$\mathrm{C}$ & 2 & 0.36515 & 0.99961 & 1.61577 & 0.01947 & 2.63485 \\
$\mathrm{C}$ & 3 & 0.36497 & 0.99961 & 1.61594 & 0.01948 & 2.63503 \\
$\mathrm{C}$ & 4 & 0.34811 & 0.99968 & 1.64310 & 0.00910 & 2.65189 \\
$\mathrm{H}$ & 5 & 0.08820 & 0.00000 & 0.41070 & 0.00110 & 0.41180 \\
$\mathrm{H}$ & 6 & 0.08820 & 0.00000 & 0.41070 & 0.00110 & 0.41180 \\
$\mathrm{O}$ & 7 & -0.25863 & 0.99987 & 3.24481 & 0.01394 & 4.25863 \\
$\mathrm{O}$ & 8 & -0.25856 & 0.99987 & 3.24475 & 0.01394 & 4.25856 \\
$\mathrm{C}$ & 9 & -0.05859 & 0.99941 & 2.04863 & 0.01054 & 3.05859 \\
$\mathrm{C}$ & 10 & -0.08474 & 0.99958 & 2.07625 & 0.00890 & 3.08474 \\
$\mathrm{C}$ & 11 & -0.05599 & 0.99941 & 2.04646 & 0.01012 & 3.05599 \\
$\mathrm{C}$ & 12 & -0.07207 & 0.99942 & 2.06200 & 0.01065 & 3.07207 \\
$\mathrm{C}$ & 13 & -0.08796 & 0.99958 & 2.07901 & 0.00937 & 3.08796 \\
$\mathrm{C}$ & 14 & -0.07760 & 0.99951 & 2.06880 & 0.00928 & 3.07760 \\
$\mathrm{H}$ & 15 & 0.11725 & 0.00000 & 0.38124 & 0.00150 & 0.38275 \\
$\mathrm{H}$ & 16 & 0.10961 & 0.00000 & 0.38947 & 0.00092 & 0.39039 \\
$\mathrm{H}$ & 17 & 0.11738 & 0.00000 & 0.38112 & 0.00150 & 0.38262 \\
$\mathrm{H}$ & 18 & 0.10967 & 0.00000 & 0.38936 & 0.00096 & 0.39033 \\
\hline Total & & 0.50004 & 11.99519 & 28.85311 & 0.15166 & 40.99996 \\
\hline & & & & & &
\end{tabular}


Supporting Information

${ }^{1} \mathrm{H}$ NMR (500 MHz, $\mathrm{CDCl}_{3}$ ) of $\mathbf{5 a a}$

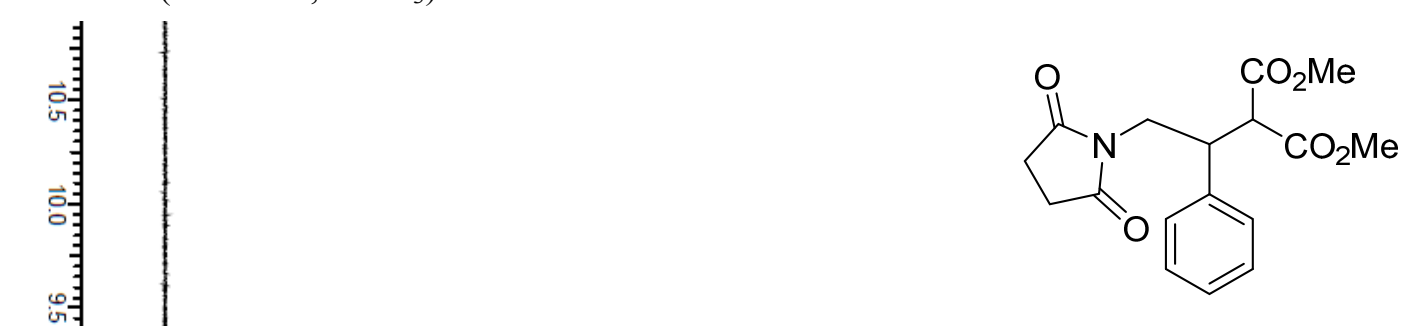

䏬

is

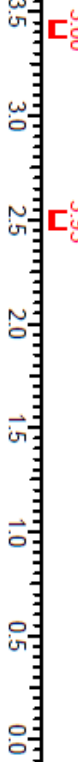

$\left[\begin{array}{r}7.2814 \\ -7.2636 \\ -7.2533 \\ -7.2373 \\ -7.2344 \\ -7.2201 \\ -7.1961 \\ 7.1823\end{array}\right.$

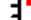


Supporting Information

${ }^{13} \mathrm{C} \mathrm{NMR} \mathrm{(500} \mathrm{MHz,} \mathrm{CDCl}_{3}$ ) of $\mathbf{5 a a}$

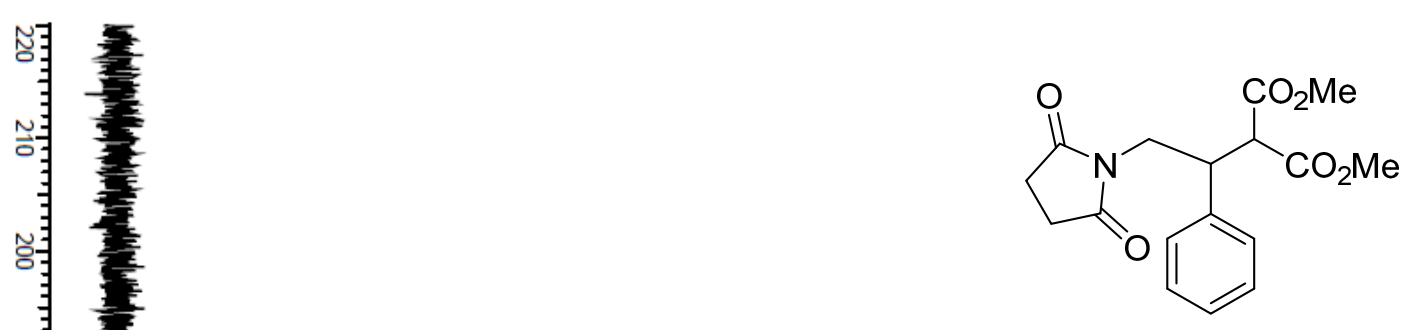

용

趾

항

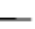

항

호

홍

8

果

ช

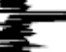

$=-$

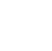

$-176.8091$

$-168.3105$

ᄂ 167.5665

$-137.4349$

28.4498

$-127.7439$

77.2575

$-77.0000$

$-76.7520$

\section{웅}

$-56.1778$

$-52.8489$

52.4293

\urcorner$_{41.8894}^{42.4426}$

$-27.8585$ 
Supporting Information

${ }^{1} \mathrm{H} \mathrm{NMR}\left(500 \mathrm{MHz}, \mathrm{CDCl}_{3}\right)$ of $\mathbf{5 b a}$

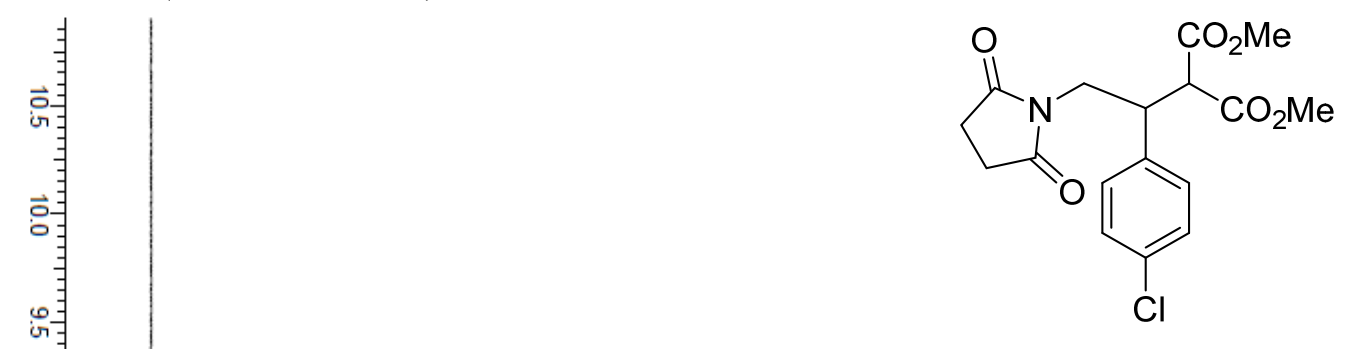

\%

i

$\stackrel{\infty}{\circ}$

i

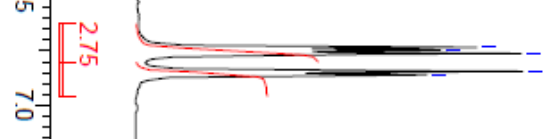

7.2665

-7.2533
-7.2373

-7.1554

$-7.1554$

in

i

g

G

영

i

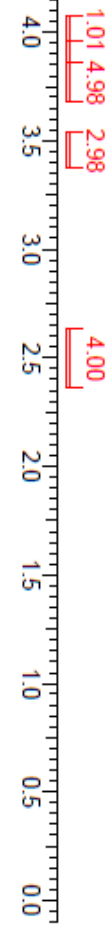
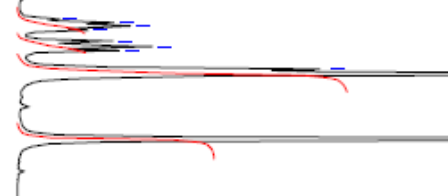
Supporting Information

${ }^{13} \mathrm{C} \mathrm{NMR}\left(500 \mathrm{MHz}, \mathrm{CDCl}_{3}\right)$ of $\mathbf{5 b a}$

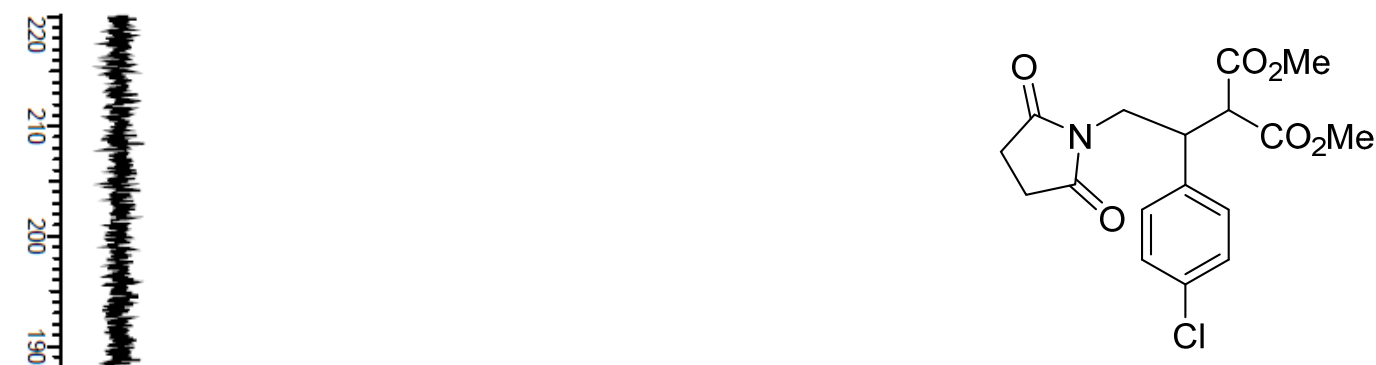

$-176.7423$

$\check{\digamma}^{168.0243}$

$-135.9755$

$-133.6100$

129.7565
-128.6596

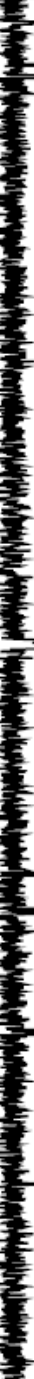

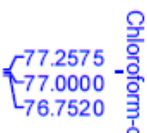

$-56.0443$

52.9157

52.562

$T^{41.8512}$

$-41.6032$

$-27.8680$ 
Supporting Information

${ }^{1} \mathrm{H}$ NMR $\left(500 \mathrm{MHz}, \mathrm{CDCl}_{3}\right)$ of $\mathbf{5} \mathbf{c a}$
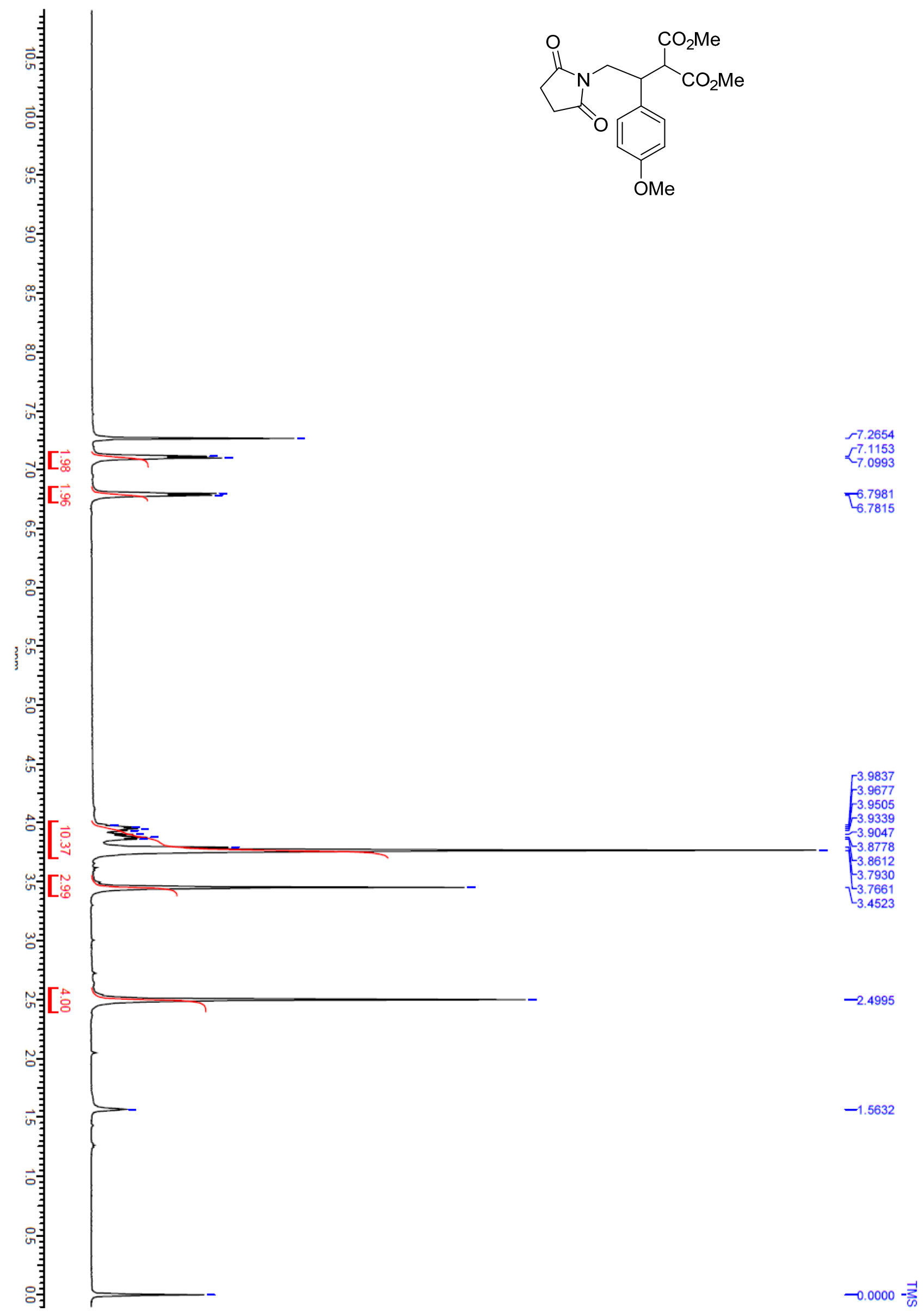
Supporting Information

${ }^{13} \mathrm{C} \mathrm{NMR}\left(500 \mathrm{MHz}, \mathrm{CDCl}_{3}\right)$ of $\mathbf{5} \mathbf{c a}$

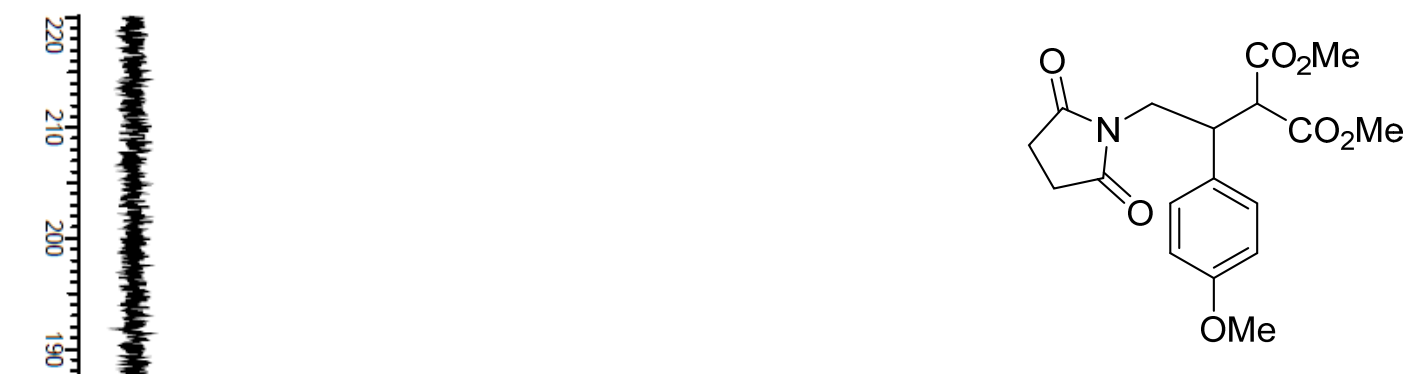

$-176.8186$

$\complement^{168.3486}$

$-158.8961$

$\tau_{129.2319}^{129.4131}$

$-113.7512$

宗

$-$

홍

$8:$

o:

77.2575

$-77.0000$

$-76.7425$

하

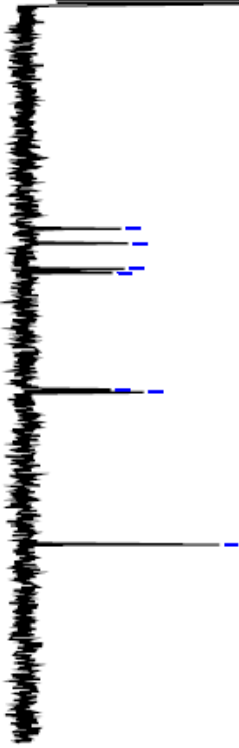

$-27.8680$

$-56.4067$

$-55.1095$

$\tau_{52.4293}^{52.7917}$

$\Gamma_{41.6605}^{41.9085}$ 
Supporting Information

${ }^{1} \mathrm{H}$ NMR $\left(500 \mathrm{MHz}, \mathrm{CDCl}_{3}\right)$ of $\mathbf{5 d a}$

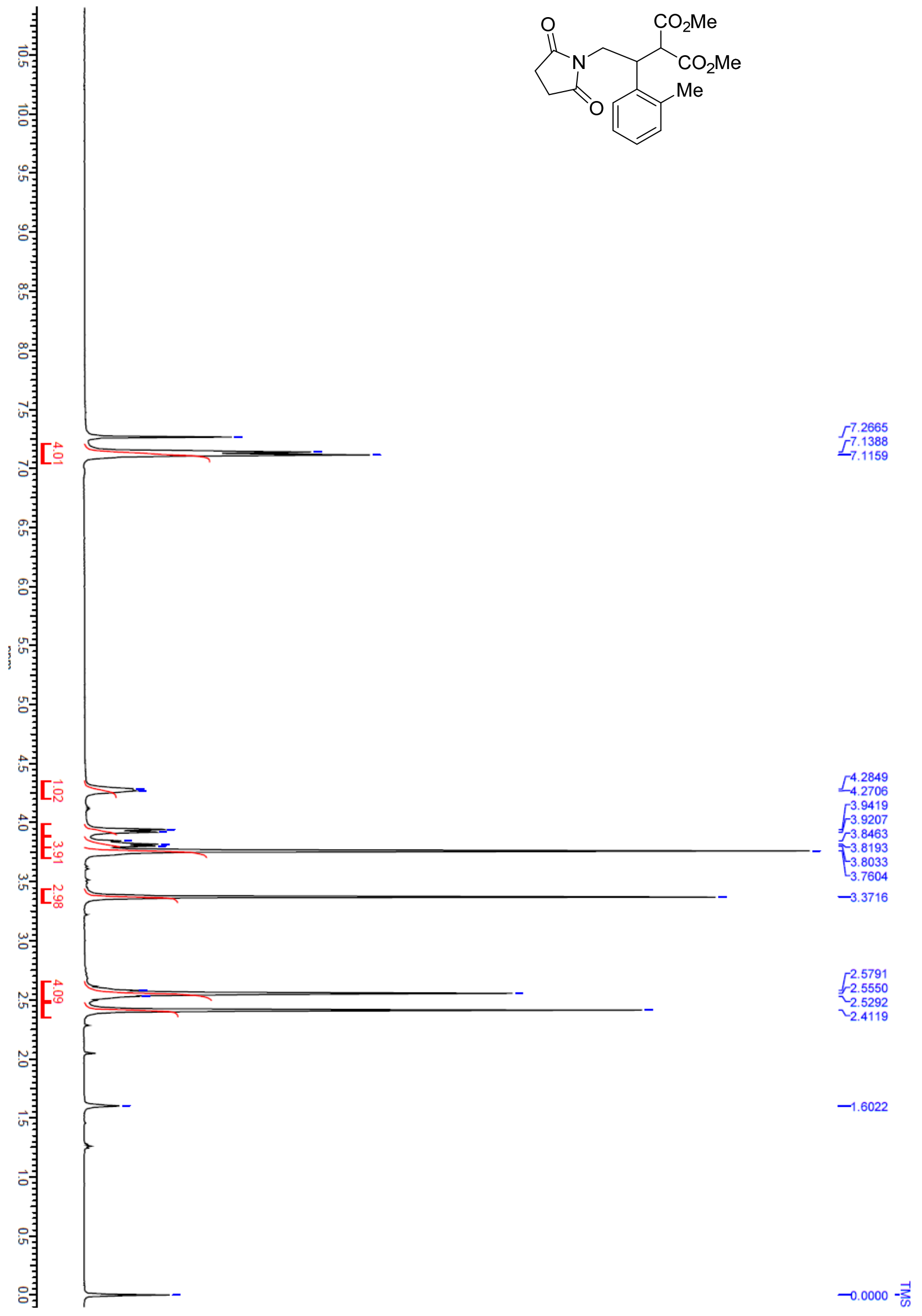




\section{Supporting Information}

${ }^{13} \mathrm{C}$ NMR $\left(500 \mathrm{MHz}, \mathrm{CDCl}_{3}\right)$ of $\mathbf{5 d a}$
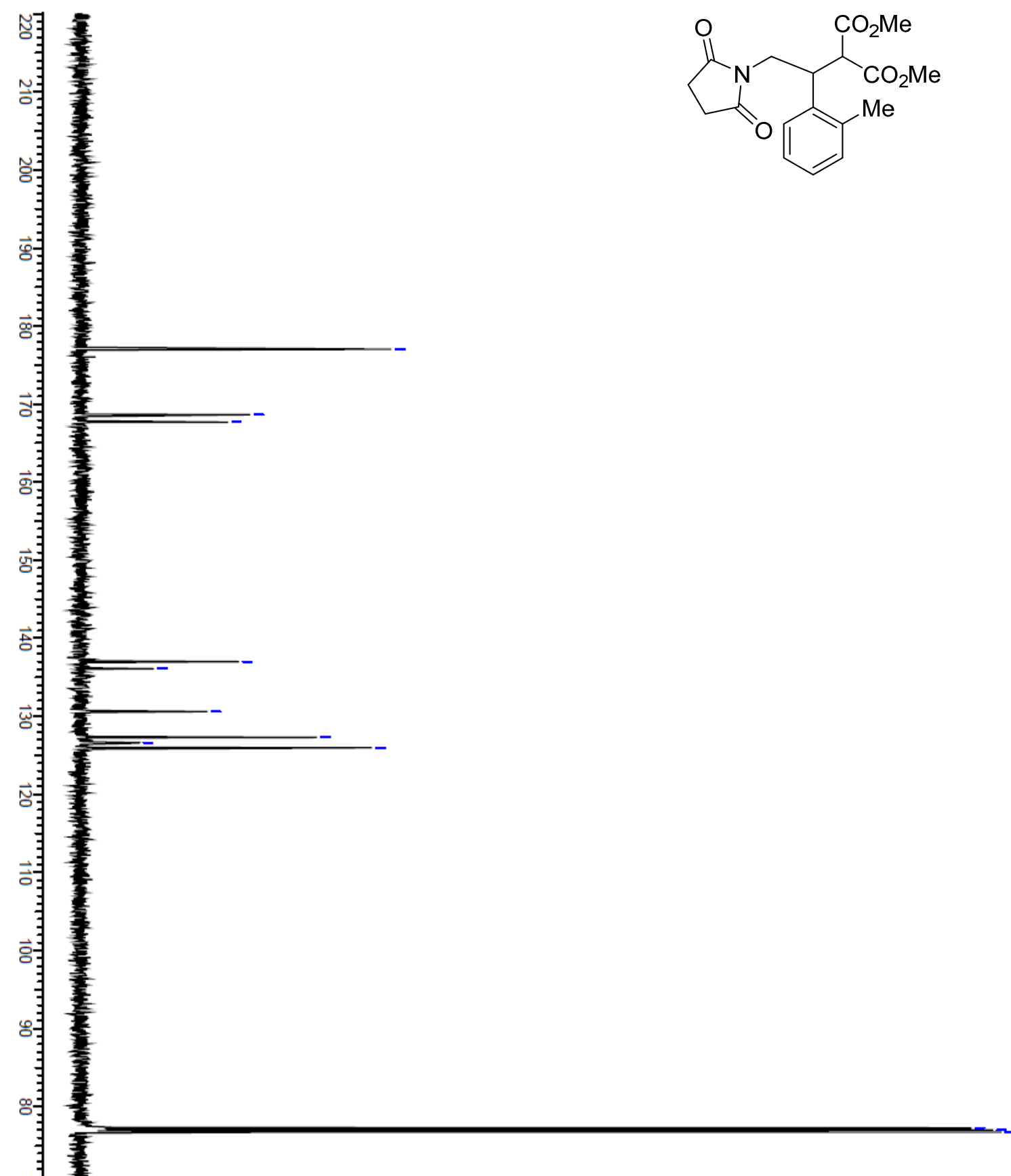
Supporting Information

${ }^{1} \mathrm{H}$ NMR $\left(500 \mathrm{MHz}, \mathrm{CDCl}_{3}\right)$ of $\mathbf{5 e a}$

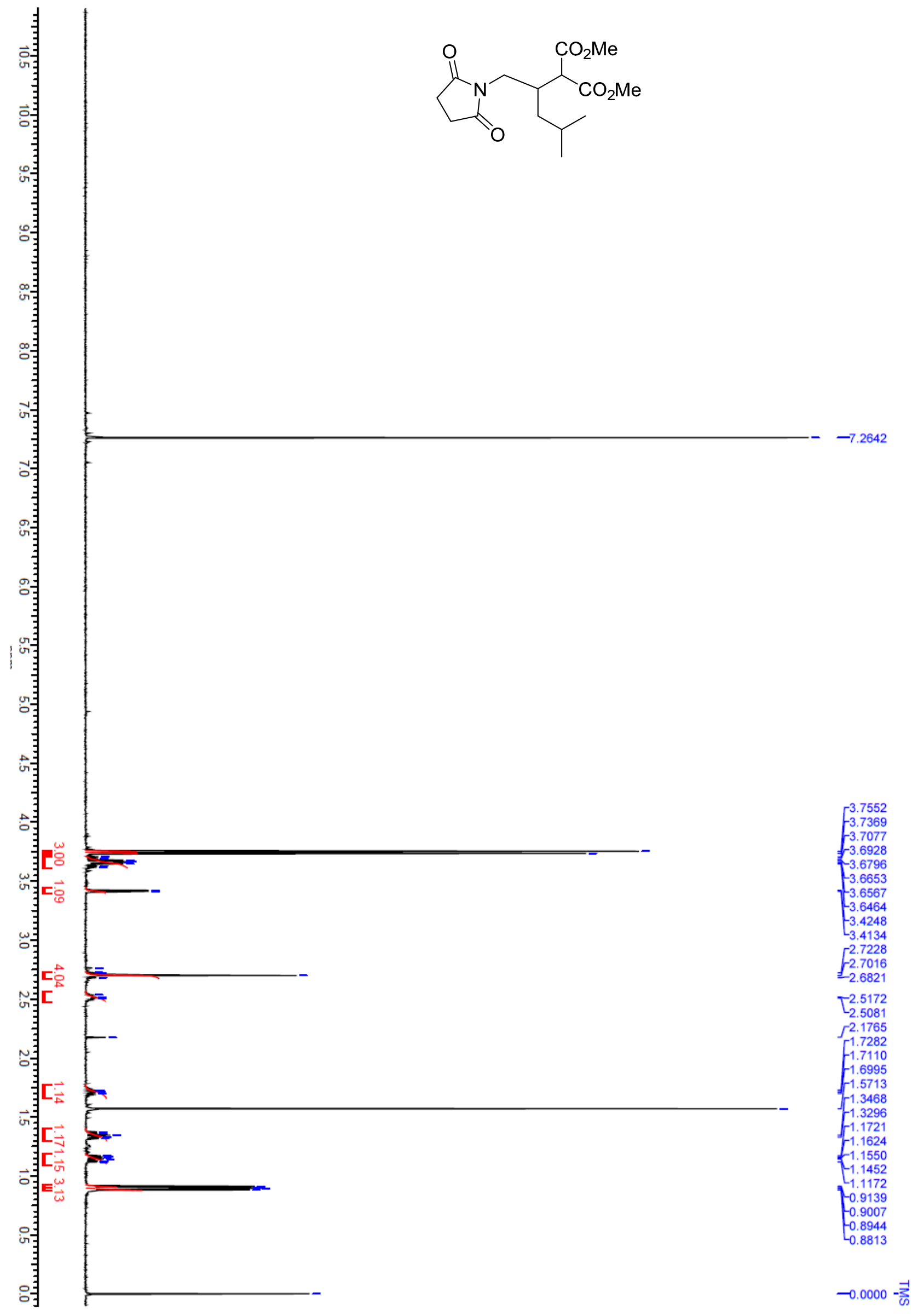


Supporting Information
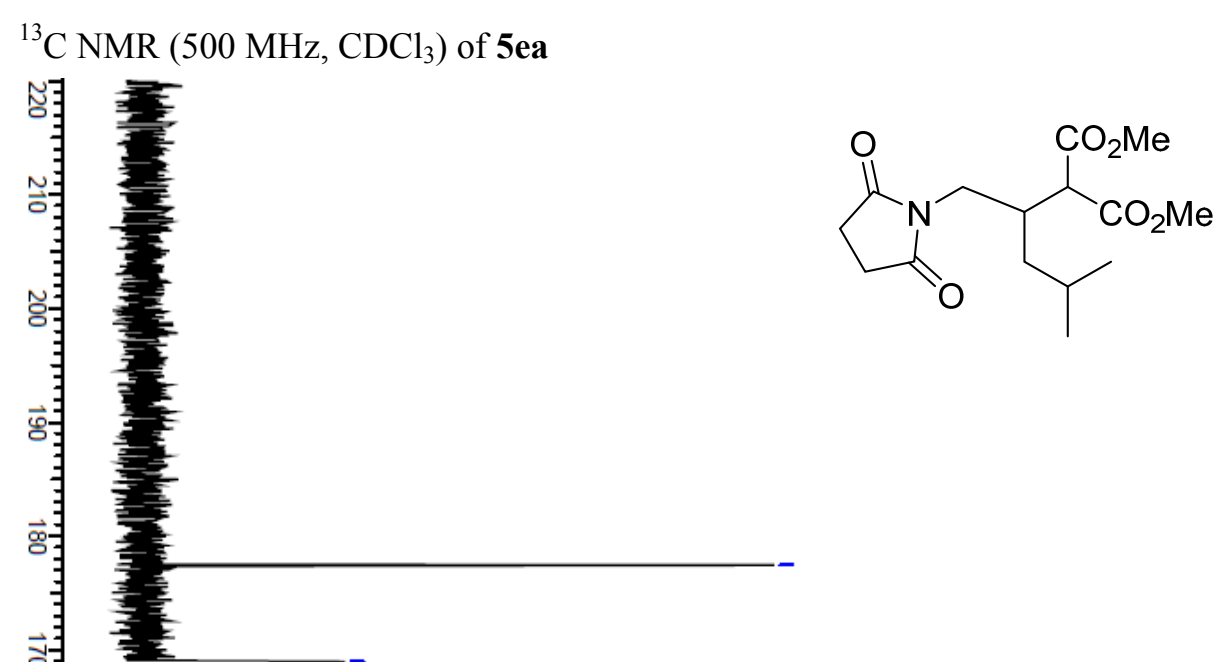
Supporting Information

${ }^{1} \mathrm{H} \mathrm{NMR}\left(500 \mathrm{MHz}, \mathrm{CDCl}_{3}\right)$ of $\mathbf{5 a b}$

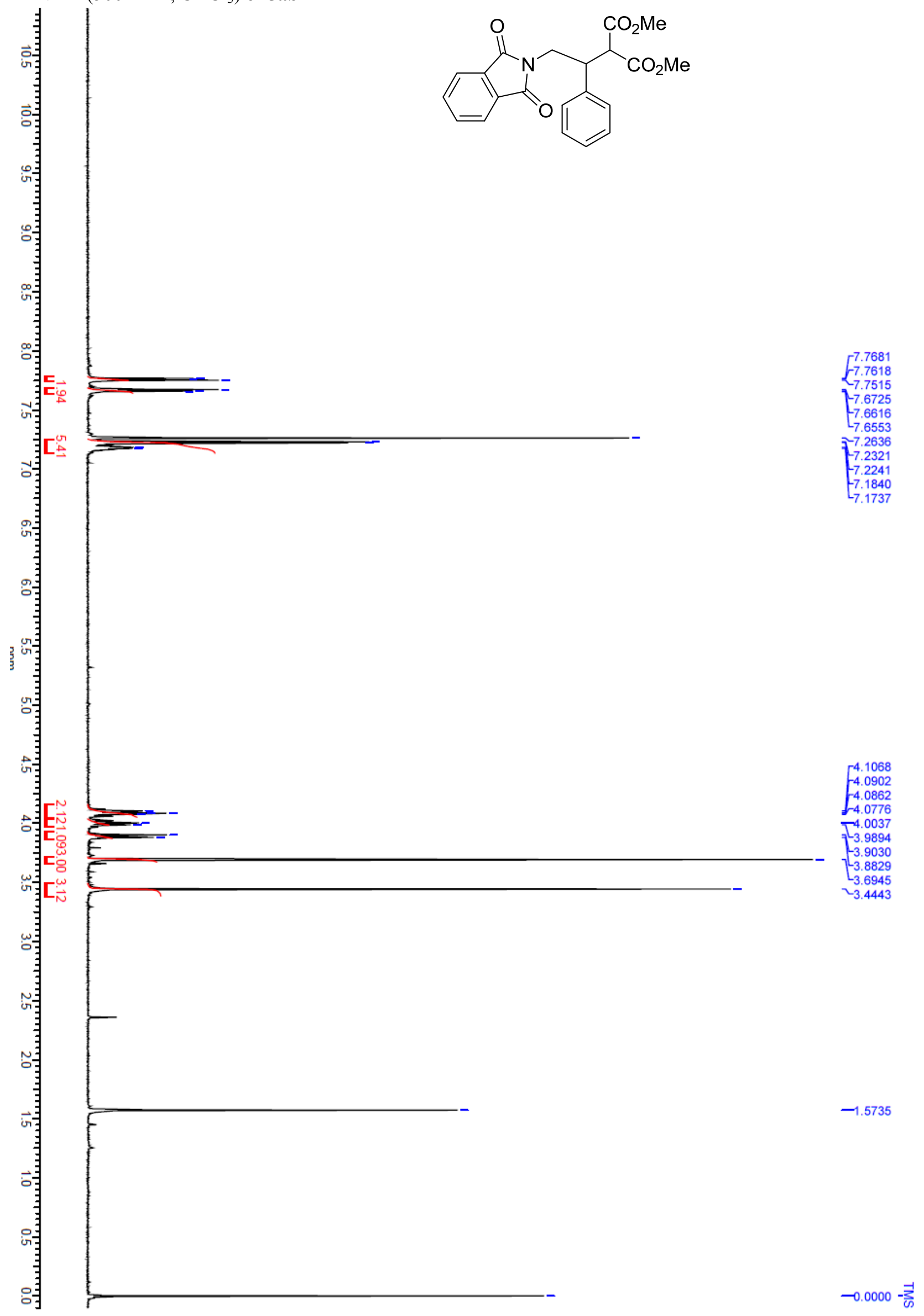


Supporting Information

$\left.{ }^{13} \mathrm{C} \mathrm{NMR} \mathrm{(500} \mathrm{MHz,} \mathrm{CDCl}_{3}\right)$ of $\mathbf{5 a b}$

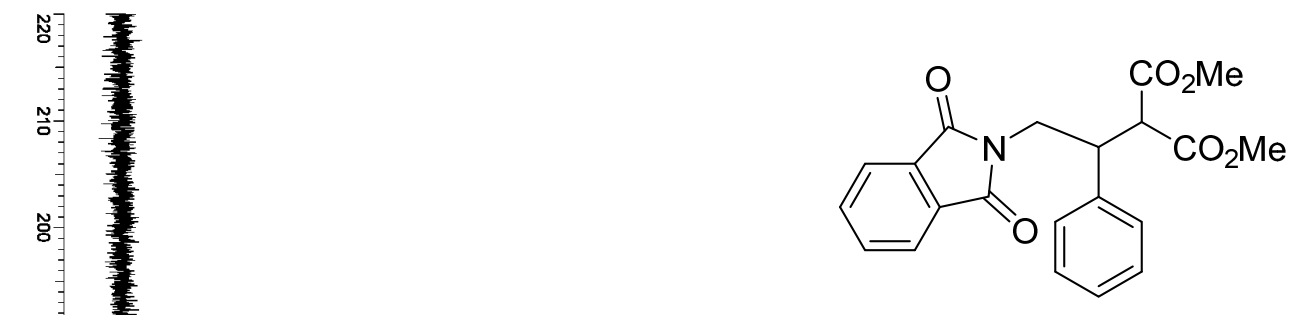

$\overrightarrow{\mathrm{g}}$

เิ

168.2055459 $\leftarrow_{167.6237081}^{167.8621611}$

$-137.5493404$

$-133.8961564$ 128.4402335

${ }_{-128.3543873}^{128.440335}$

$-127.6390127$

$-123.1846158$

$\vec{\nabla}$

꽄

$\overrightarrow{\mathrm{o}}$

8

8
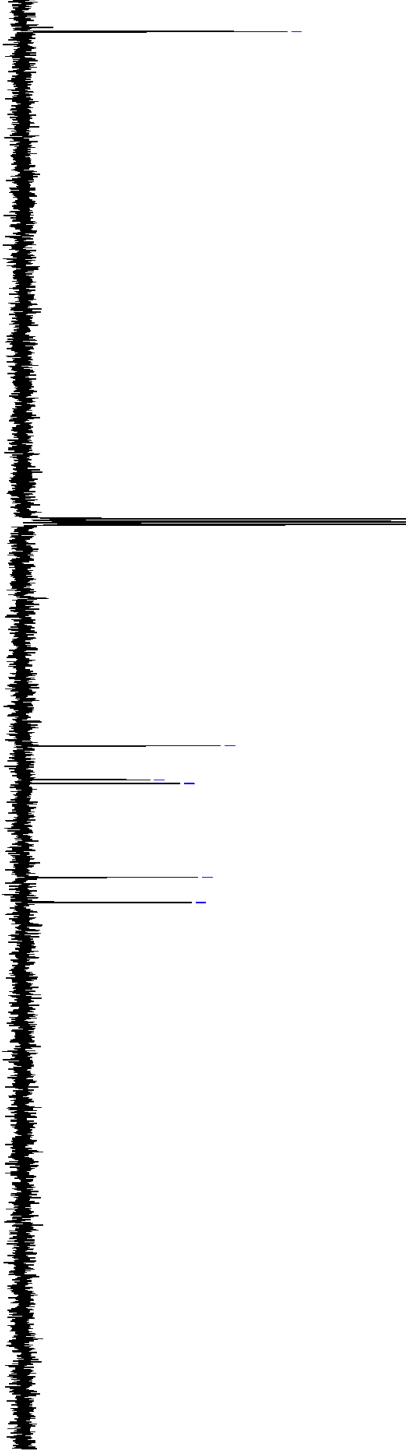

772575487

770000101

76.7424716 产

55.9775237

${ }_{-52.4292637}^{52.7726446}$

$-43.6158432$

$-41.2884921$ 
Supporting Information

${ }^{1} \mathrm{H}$ NMR $\left(500 \mathrm{MHz}, \mathrm{CDCl}_{3}\right)$ of $\mathbf{5 b b}$

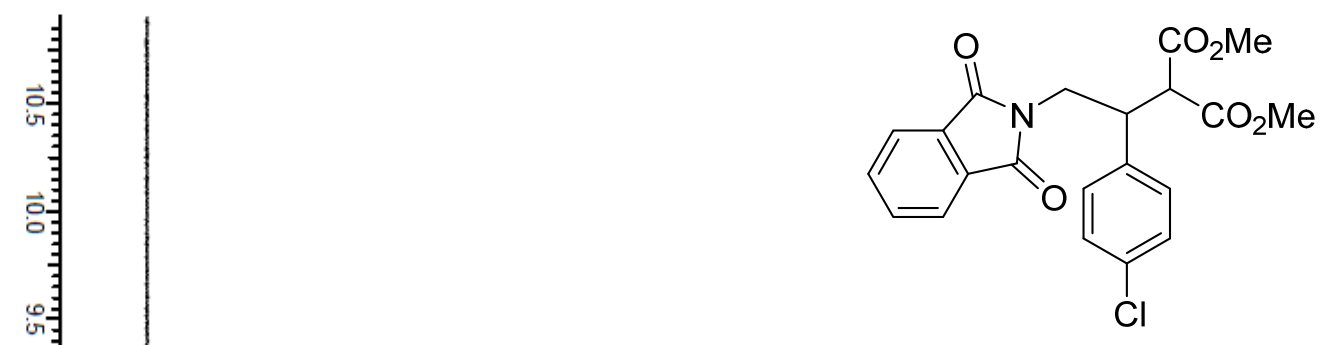

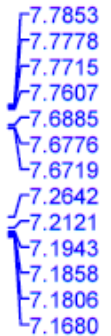

4.1217
-4.1051
-4.0942
-4.0816
-4.0747
-3.9774
-3.9705
-3.9562
-3.8405
-3.8205
-3.7117
3.4884

$-2.3575$

$-1.5810$

$-1.4401$ 
Supporting Information

${ }^{13} \mathrm{C}$ NMR (125 MHz, $\mathrm{CDCl}_{3}$ ) of $\mathbf{5 b b}$

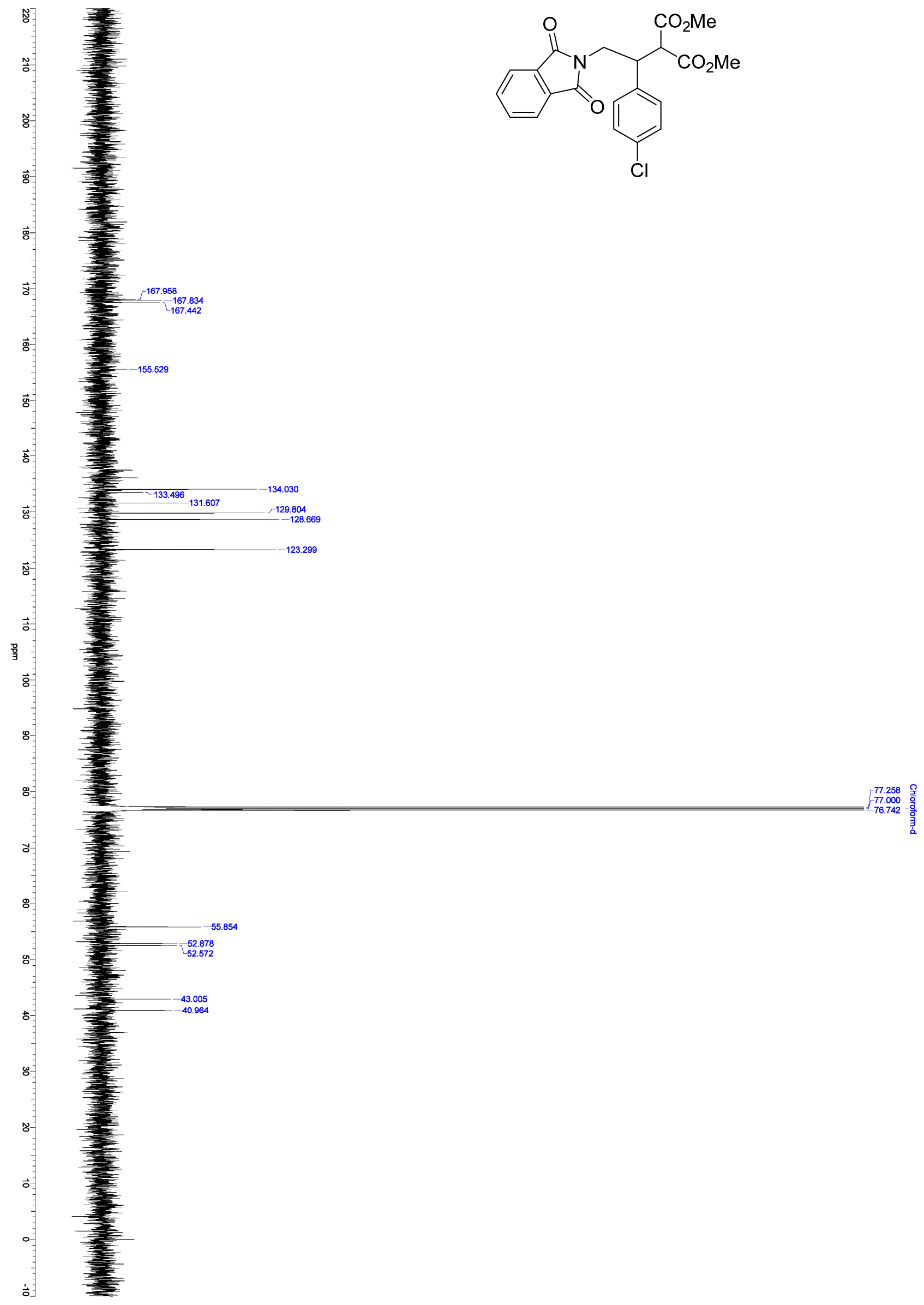


Supporting Information

${ }^{1} \mathrm{H}$ NMR $\left(500 \mathrm{MHz}, \mathrm{CDCl}_{3}\right)$ of $\mathbf{5 c b}$

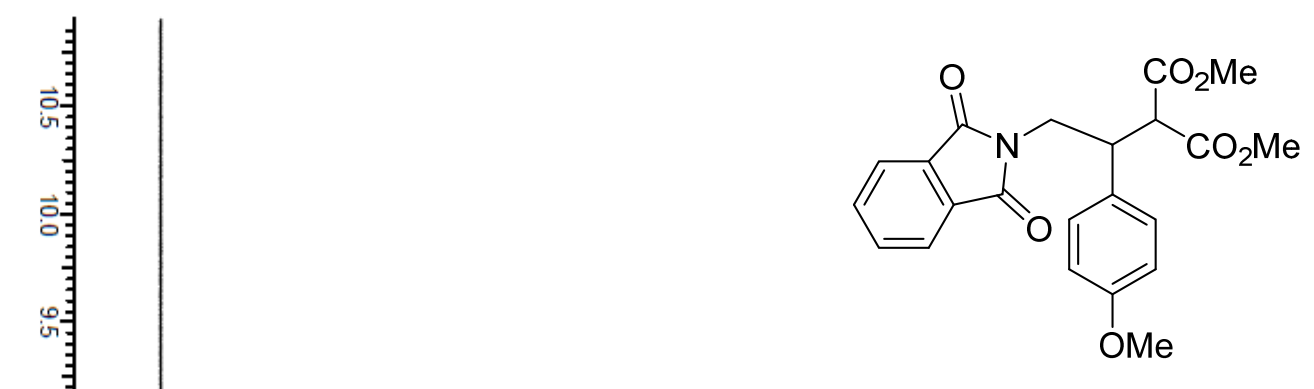

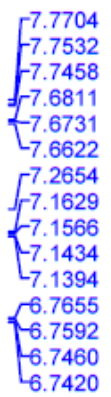

4.0988

$-4.0822$

$-4.0587$

$-4.0490$

$-3.9671$

-3.9533
-3.8388

$-3.7260$

$-3.6865$

$-3.4912$

$\omega$

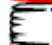

$-3.4660$

$-2.3569$

$-1.6010$

-0.0000 - 
Supporting Information

${ }^{13} \mathrm{C} \mathrm{NMR}\left(125 \mathrm{MHz}, \mathrm{CDCl}_{3}\right)$ of $\mathbf{5} \mathbf{c b}$

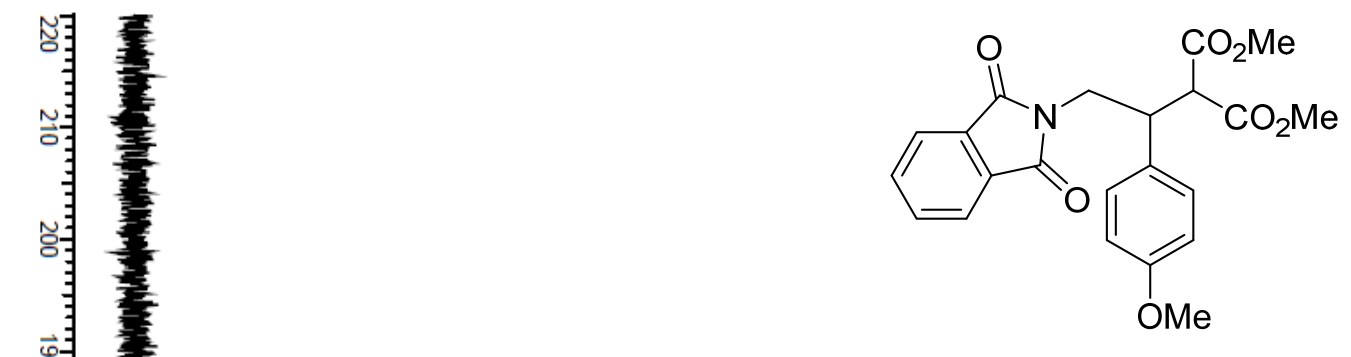

举

浬

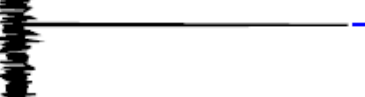

$\tau^{-168.2532}$

-167.8812
167.6809

$-158.8007$



鲜

8

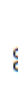

青涪

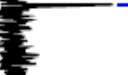

영

胡

每

$-133.8771$

$-131.7310$

$-129.4418$

$-123.1846$

$-113.7703$

77.2480

$-77.0000$

$-76.7425$

56.2541

$\int_{-55.0618}^{56.2541}$

52.7345

52.4388

$-42.8051$

$-41.2789$ 
Supporting Information

${ }^{1} \mathrm{H}$ NMR (500 MHz, $\mathrm{CDCl}_{3}$ ) of $\mathbf{5 d b}$

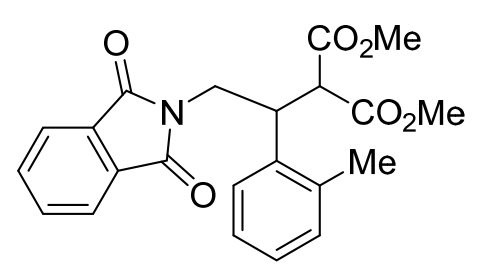

-7.7893
-7.7784
-7.6931
-7.6874
-7.6759
7.2654
-7.2069
-7.1921
-7.1222
-7.1136
-7.1067
-7.0884
7.0815

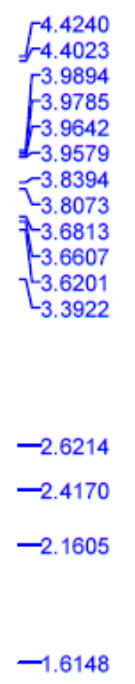

$\longrightarrow 0.0000-\stackrel{-1}{\rightleftarrows}$

S24 
Supporting Information

${ }^{13} \mathrm{C}$ NMR $\left(125 \mathrm{MHz}, \mathrm{CDCl}_{3}\right)$ of $\mathbf{5 d b}$

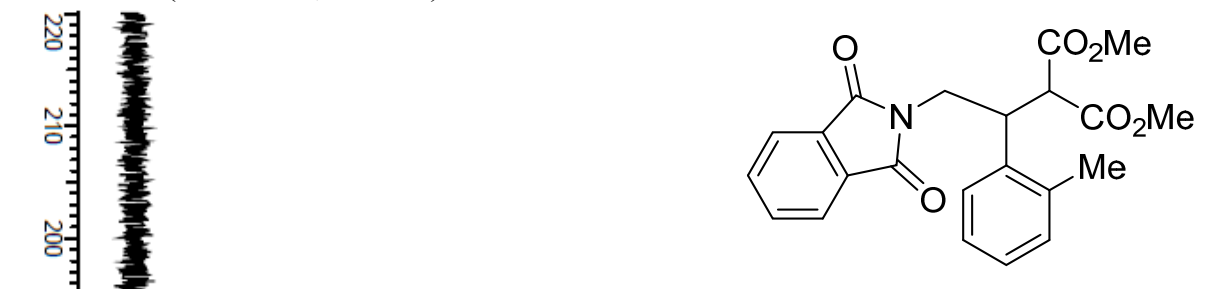

을

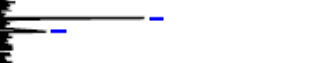

$-137.0724$

$-133.9438$

$-131.7882$

$-130.6245$

$-127.2670$

ᄂ126.0175

$\sim 123.194$ 
Supporting Information

${ }^{1} \mathrm{H}$ NMR $\left(500 \mathrm{MHz}, \mathrm{CDCl}_{3}\right)$ of $\mathbf{5 e b}$

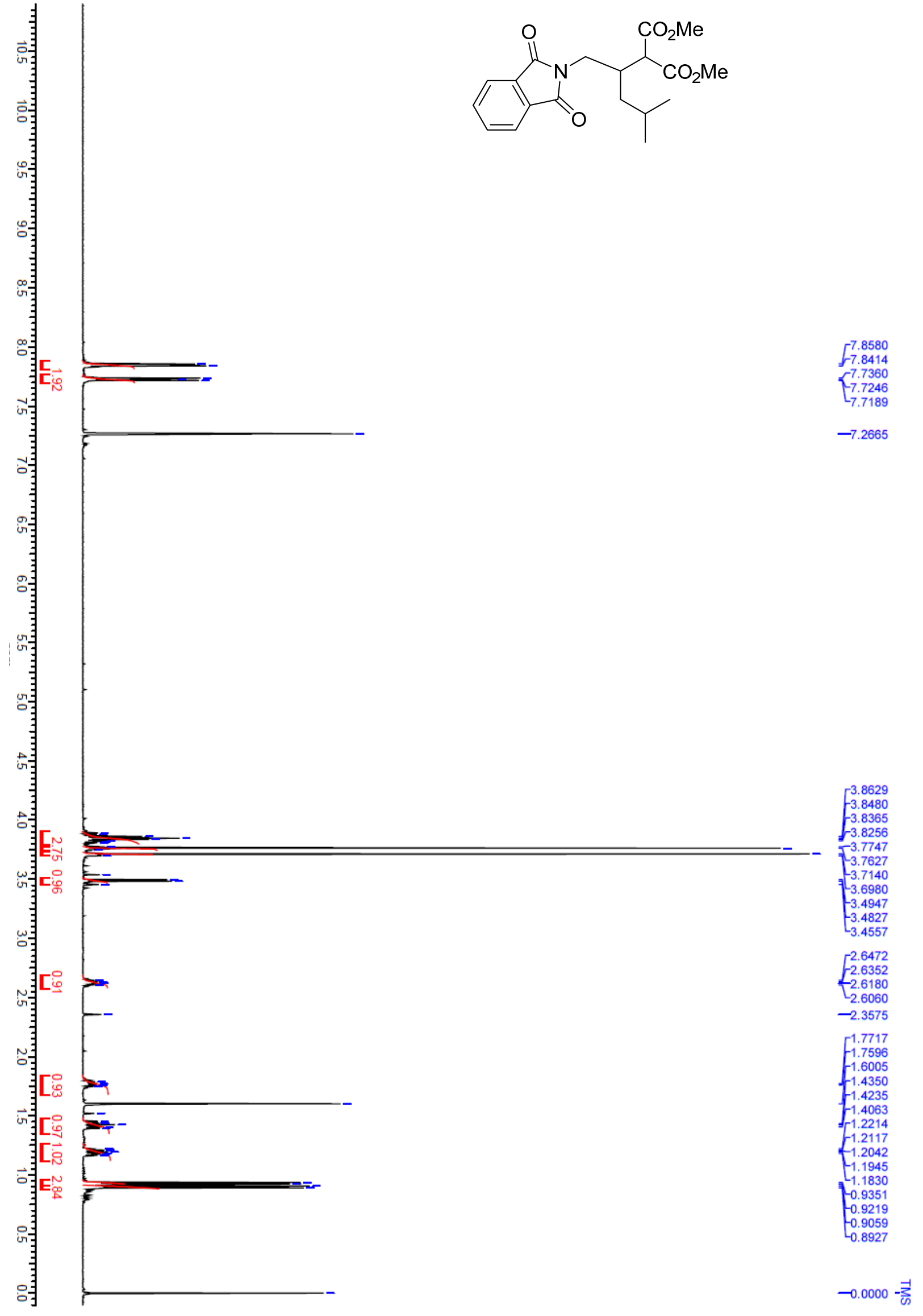


Supporting Information

${ }^{13} \mathrm{C} \mathrm{NMR}\left(125 \mathrm{MHz}, \mathrm{CDCl}_{3}\right)$ of $\mathbf{5 e b}$

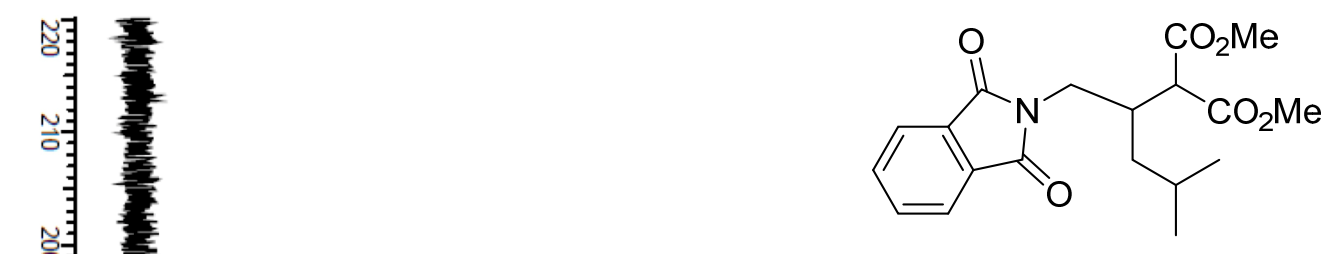

o

$\overrightarrow{\mathrm{g}}$

$\vec{\circ}$

青旁

혁

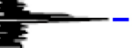

$-168.5871$

言

讅

8

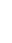

영

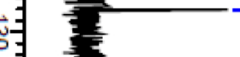

$-123.2991$

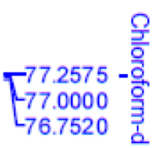

$\breve{5.4388}_{53.4308}$

$\digamma_{-38.7799}^{-39.6670}$

$-36.2236$

$-25.6075$

$-23.0893$

$-21.8970$ 
Supporting Information

${ }^{1} \mathrm{H}$ NMR (500 MHz, $\mathrm{CDCl}_{3}$ ) of $\mathbf{6 a a}$

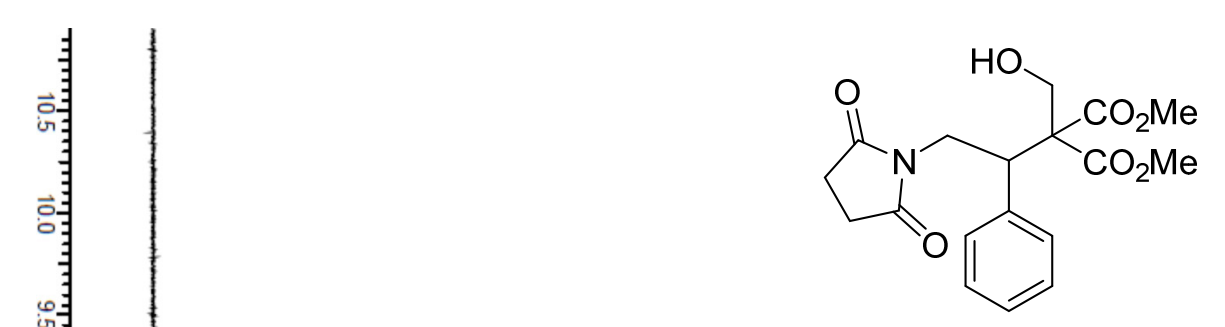

4.6244
-4.5975
-4.5752

-3.9511
-3.9425
-3.9201
-3.8720
-3.8348
-3.8113
-3.7953
3.7867




-2.4972
-2.4508
-2.4405
-2.4279
-2.4233
-2.4101
-2.3998
-2.3832
2.3735

S28 
Supporting Information

${ }^{13} \mathrm{C}$ NMR (125 MHz, $\mathrm{CDCl}_{3}$ ) of $\mathbf{6 a a}$

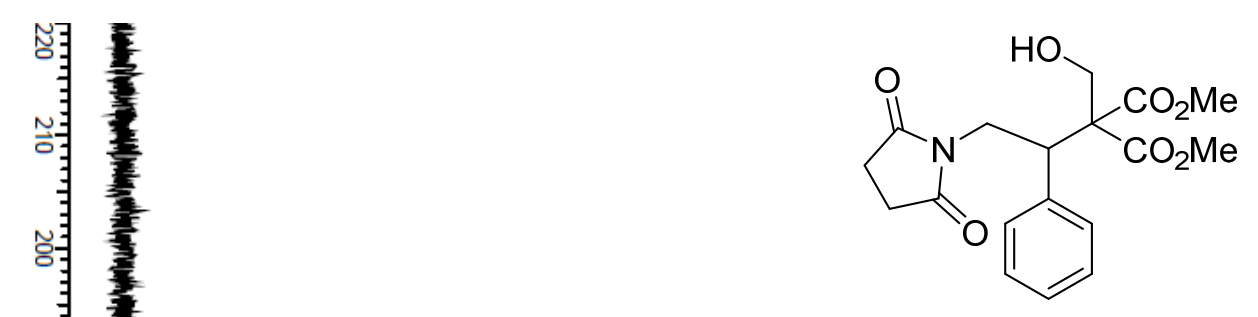

형

홍

$8:$

味

ว

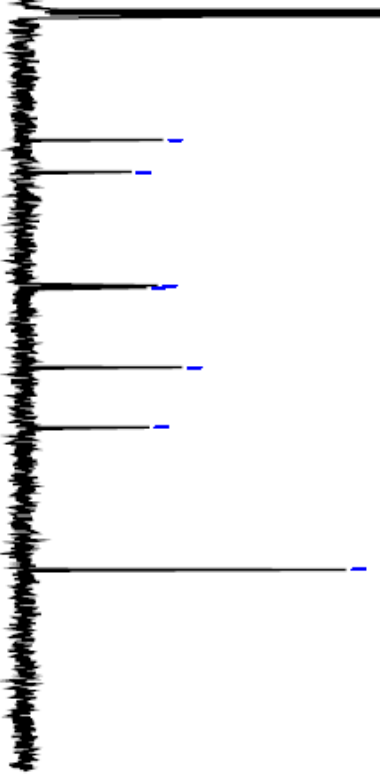

$-135.4700$

$\Upsilon^{129.3178}$

$\tau^{128.4021}$

170.4471

$-169.9797$

-128.1064

77.2575

$-77.0000$

웅

$-65.8115$

$-62.9500$

$\Gamma_{52.9443}^{52.7250}$

$-45.6666$

$-40.3633$

$-27.7536$ 
Supporting Information

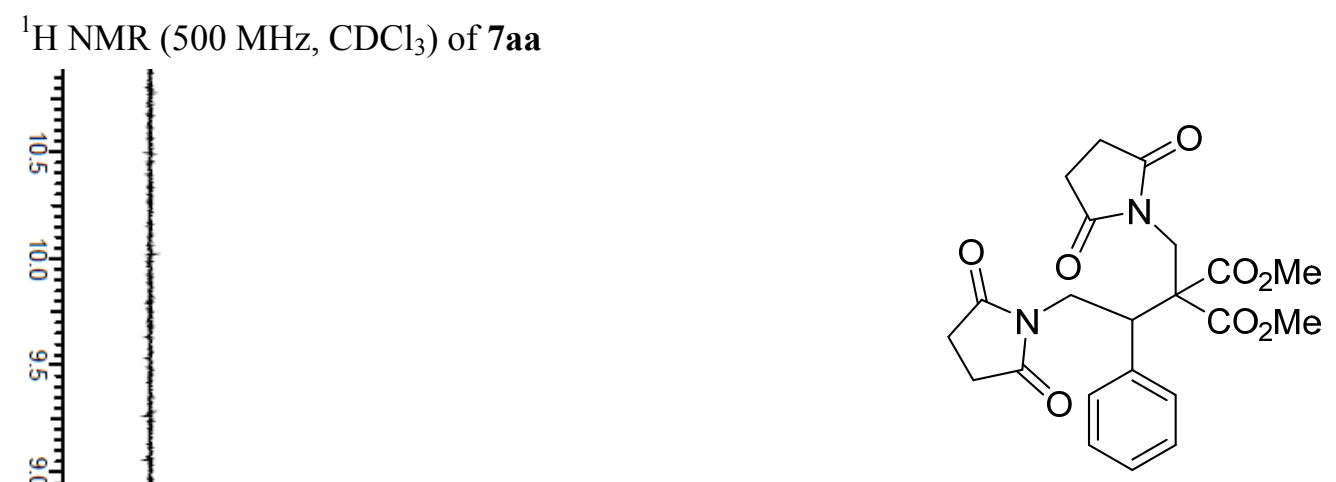

S30 
Supporting Information

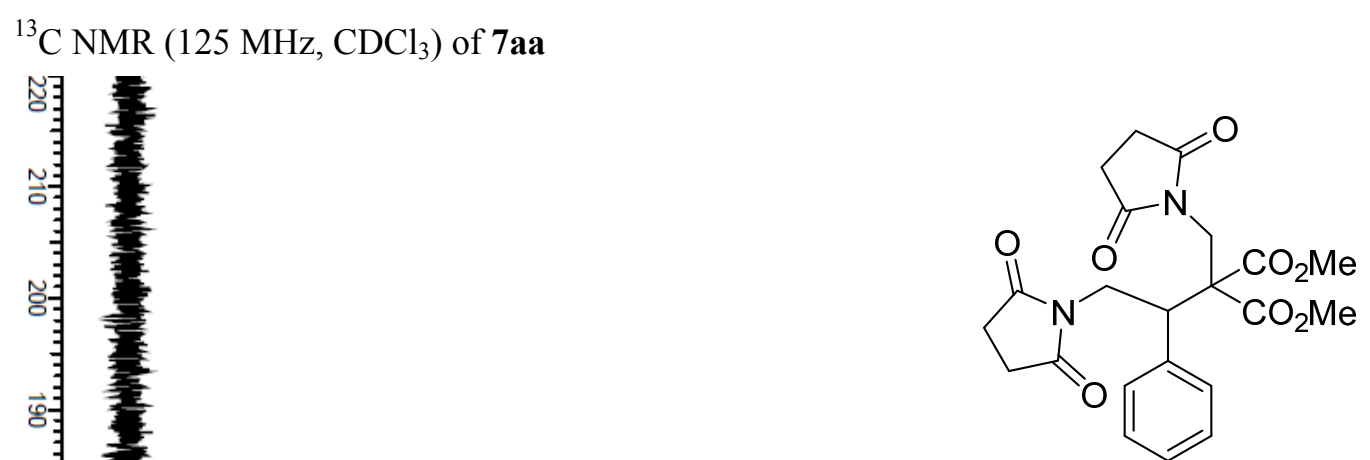


Supporting Information

${ }^{1} \mathrm{H}$ NMR $\left(500 \mathrm{MHz}, \mathrm{CDCl}_{3}\right)$ of $\mathbf{7 a b}$

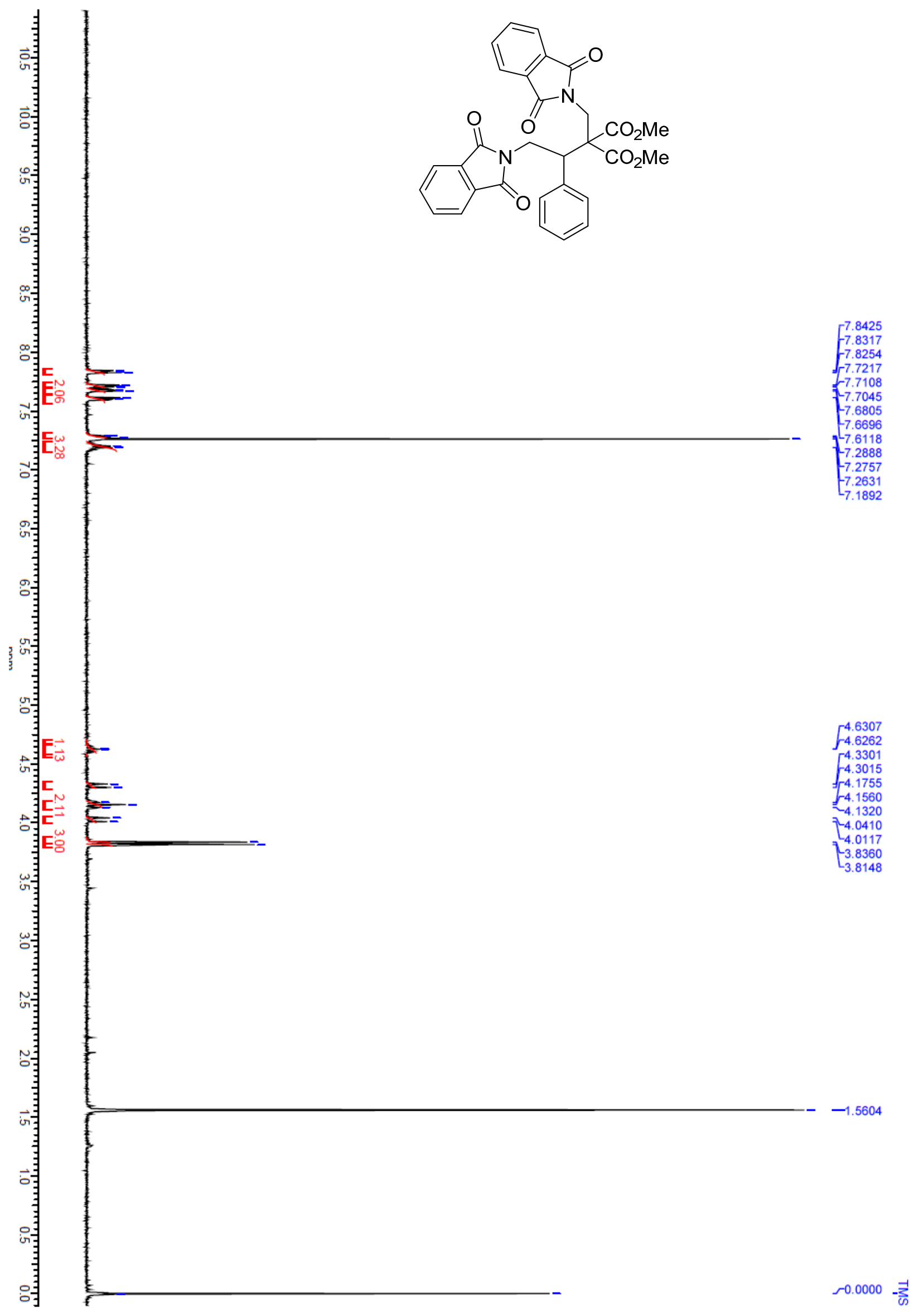


Supporting Information

${ }^{13} \mathrm{C}$ NMR $\left(125 \mathrm{MHz}, \mathrm{CDCl}_{3}\right)$ of $7 \mathbf{a b}$

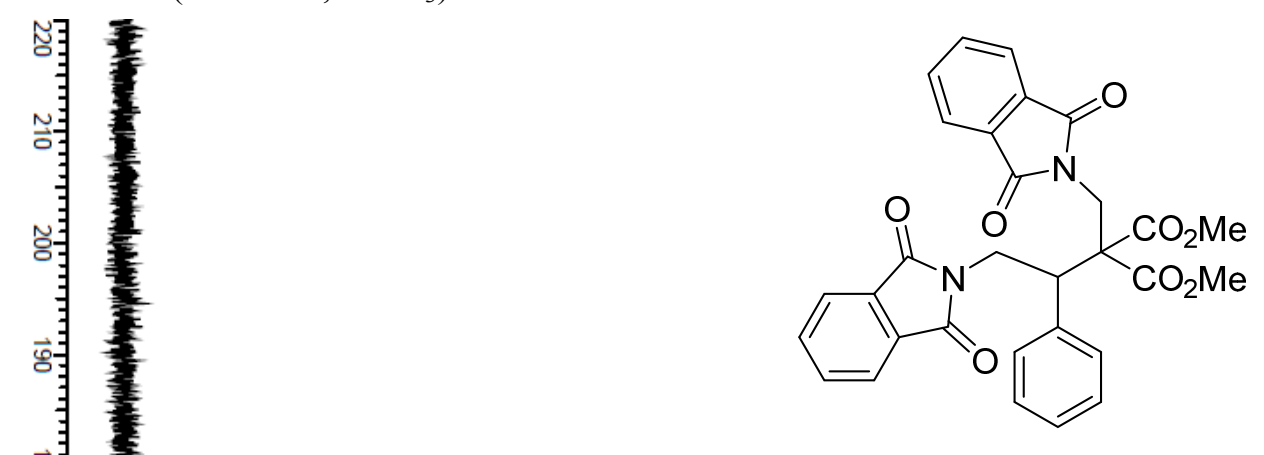

$\Gamma^{169.1880}$

$-169.0640$

167.9384

135.2792

$-134.0488$

$-133.6577$

131.8168

$L_{131.6260}$

$-129.9473$

$-128.2208$

$-128.0682$

$-123.3849$ 
Supporting Information

${ }^{1} \mathrm{H}$ NMR $\left(500 \mathrm{MHz}, \mathrm{CDCl}_{3}\right)$ of $\mathbf{7 b b}$

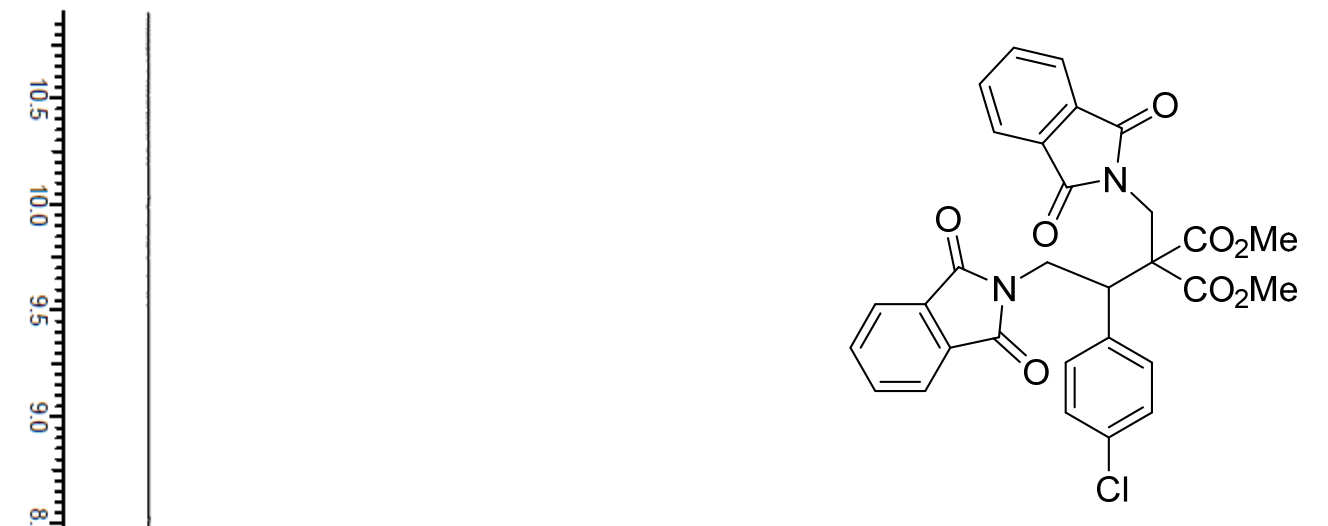

7.8511
-7.8403
-7.7389
-7.7286
-7.6920
-7.6857
-7.6341
-7.6232
-7.2963
-7.2791
-7.2671
-7.1823
7.1651

4.6313

$-4.6084$

-4.6050
-4.5821

$-4.3622$

$-4.3336$

4.1904

$-4.1675$

$-4.1526$

$-4.0369$

-4.0077
-3.8251

$-3.7976$

$-1.6222$

$-0.8217$

0.0704

-0.0000 - 궁 
Supporting Information

${ }^{13} \mathrm{C}$ NMR $\left(125 \mathrm{MHz}, \mathrm{CDCl}_{3}\right)$ of $7 \mathbf{b b}$

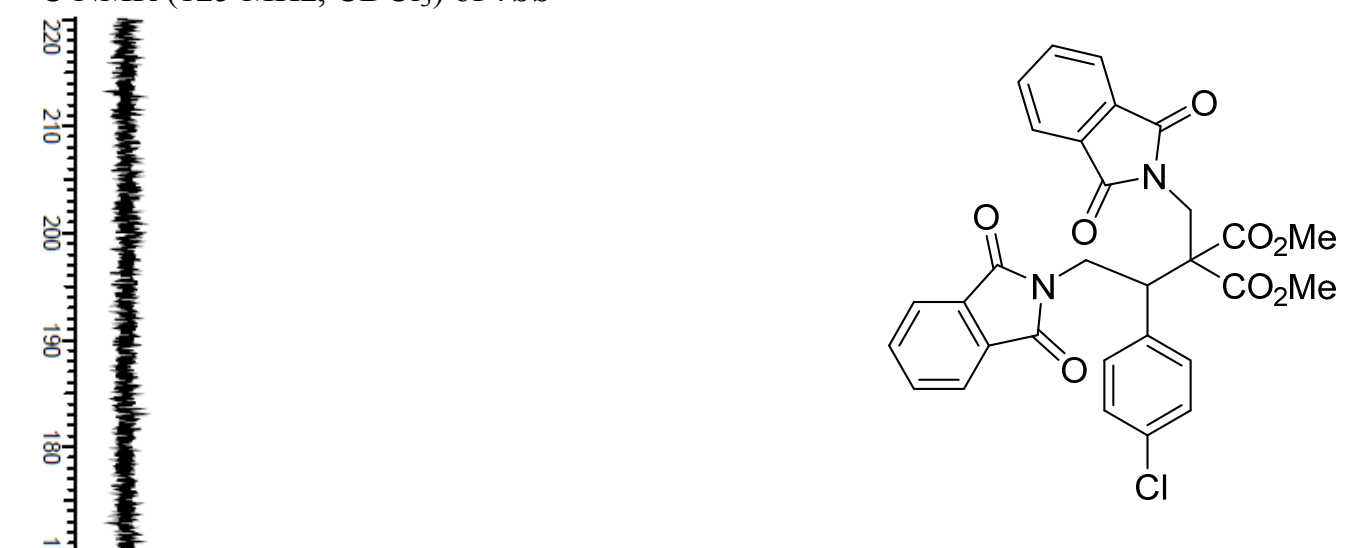

$-168.9114$ $\mathbf{L}_{167.7286}^{168.0243}$

웜

जे

흘

胡

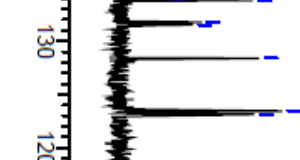

註

혁

8

\section{。}

。

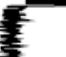

77.2575

$-77.0000$

$\leftarrow_{76.7425}$

$-133.9534$

133.8103

$-131.5402$

$-128.3735$

$\tau^{123.4612}$

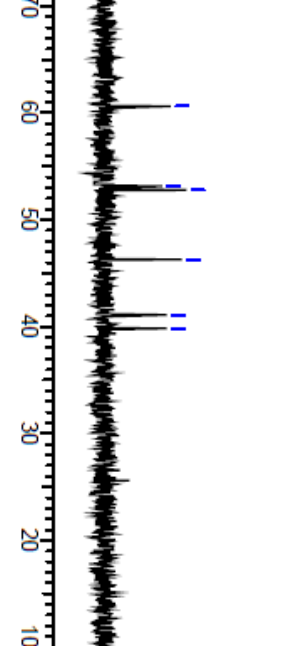

$-60.6227$

$\complement_{-52.8013}^{53.1828}$

$-46.2580$

$\neg 1.1263$

$\mathfrak{ح}^{4} 9.8196$ 
Supporting Information

${ }^{1} \mathrm{H}$ NMR $\left(500 \mathrm{MHz}, \mathrm{CDCl}_{3}\right)$ of $7 \mathbf{c b}$

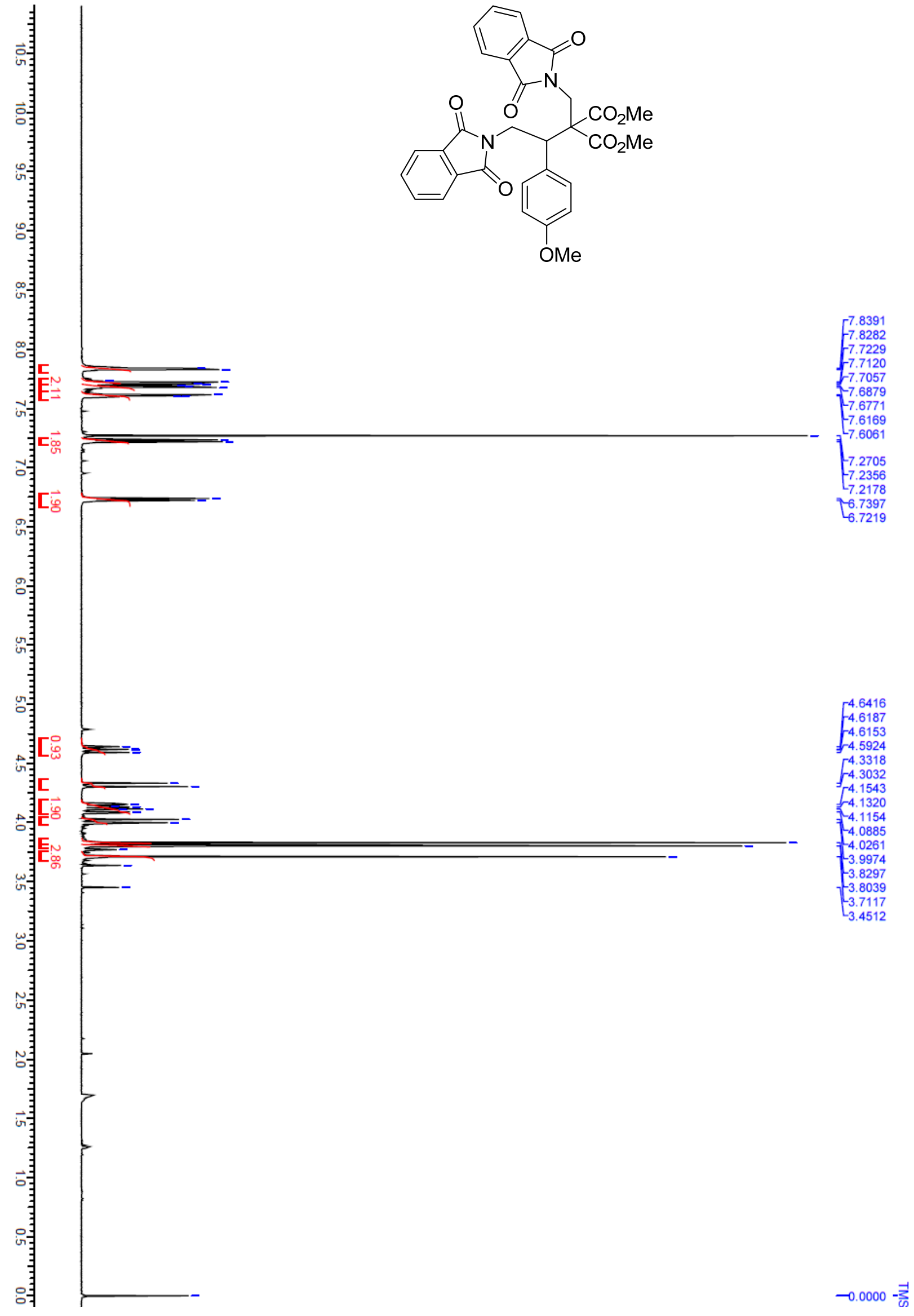


Supporting Information

${ }^{13} \mathrm{C} \mathrm{NMR}\left(125 \mathrm{MHz}, \mathrm{CDCl}_{3}\right)$ of $7 \mathbf{c b}$
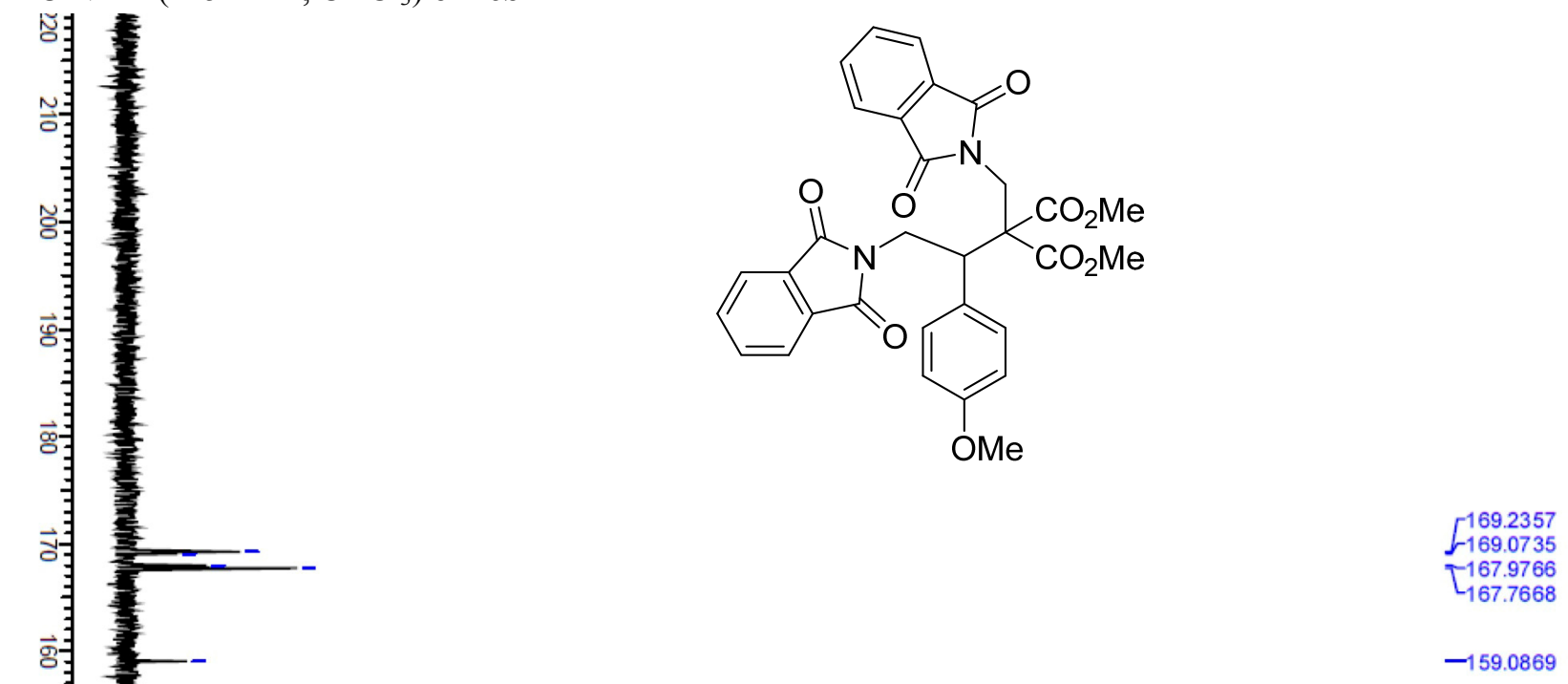

$\Sigma_{-133.6482}^{134.0297}$ $\underline{J}_{-133.6482}$ $\Gamma_{131.8359}^{131.6737}$

$-126.9332$

123.3658
-123.0225

$\tau_{123.0225}$

寒

옹

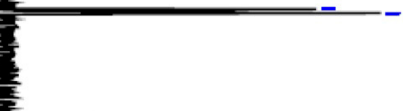

$-113.5509$

8

용

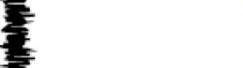

77.2575

$-77.0000-$ -

$-76.7425$

o

8

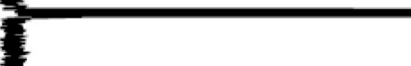

$-60.7467$

$-54.9855$

$-53.0683$

$\bigsqcup_{52.6296}$

$-46.3438$

$-41.3171$

$\checkmark 40.0104$ 
Supporting Information

${ }^{1} \mathrm{H} \mathrm{NMR}\left(500 \mathrm{MHz}, \mathrm{CDCl}_{3}\right)$ of $\mathbf{7 d b}$
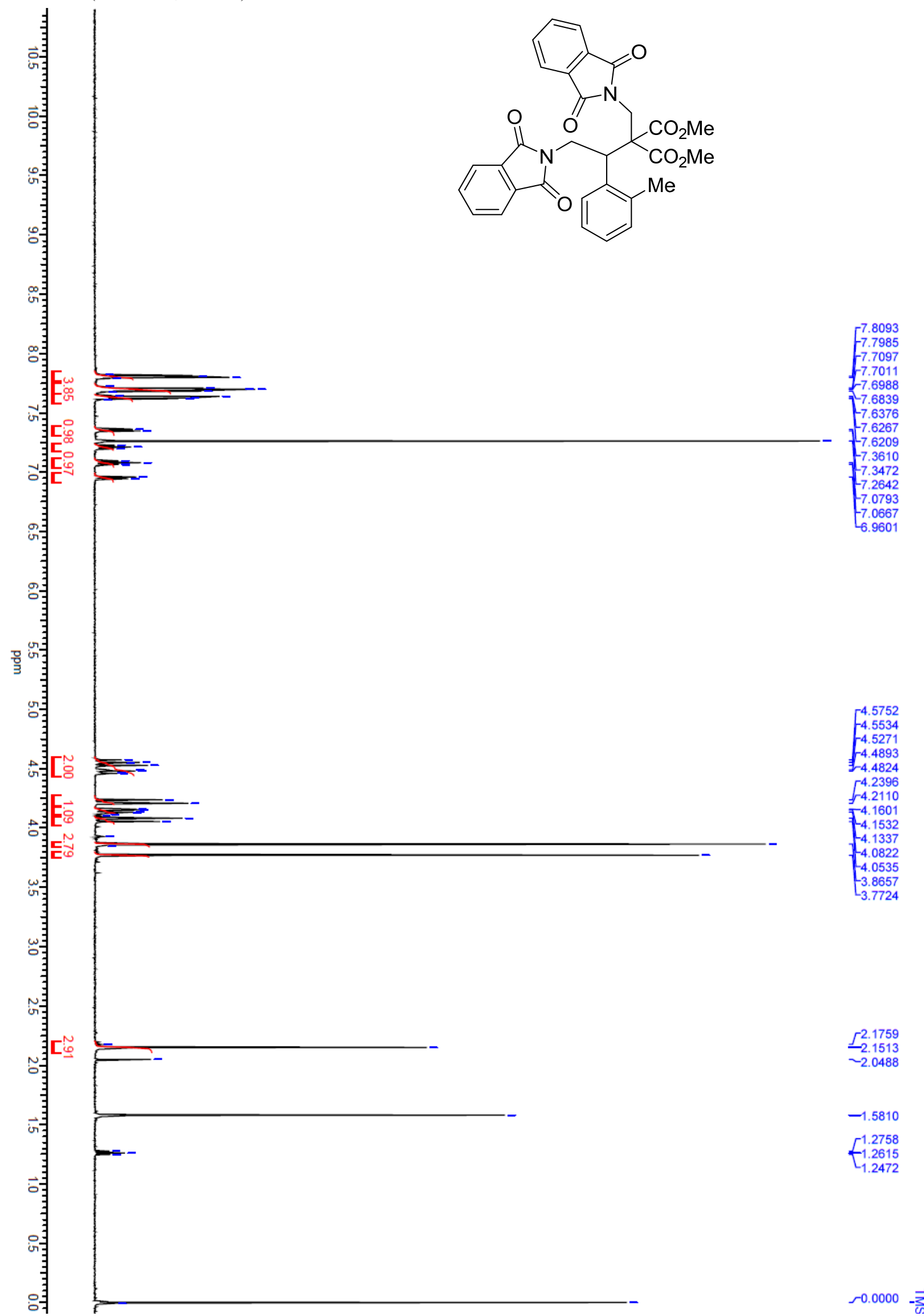
Supporting Information

${ }^{13} \mathrm{C}$ NMR (125 MHz, $\left.\mathrm{CDCl}_{3}\right)$ of $\mathbf{7 d b}$

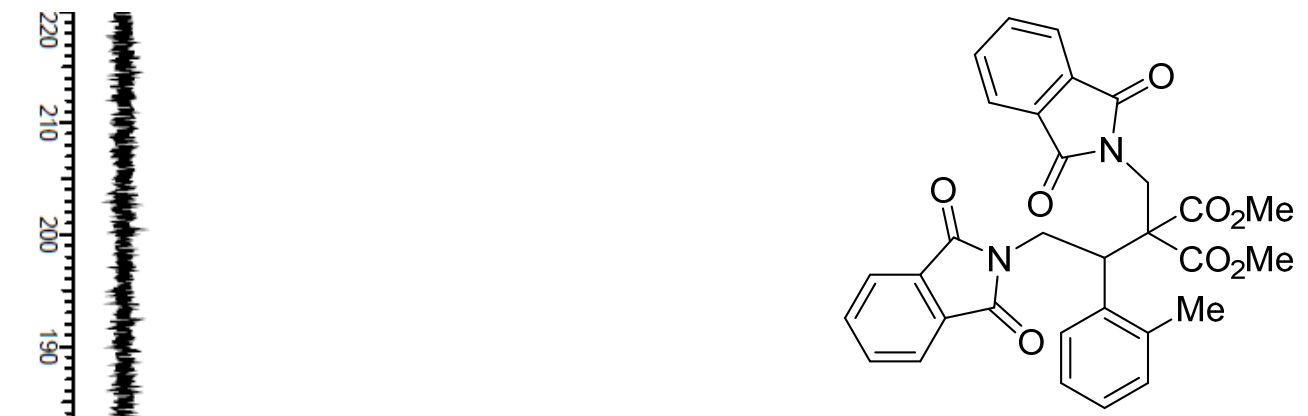

169.6935 
Supporting Information

${ }^{1} \mathrm{H}$ NMR $\left(500 \mathrm{MHz}, \mathrm{CDCl}_{3}\right)$ of $7 \mathbf{e b}$

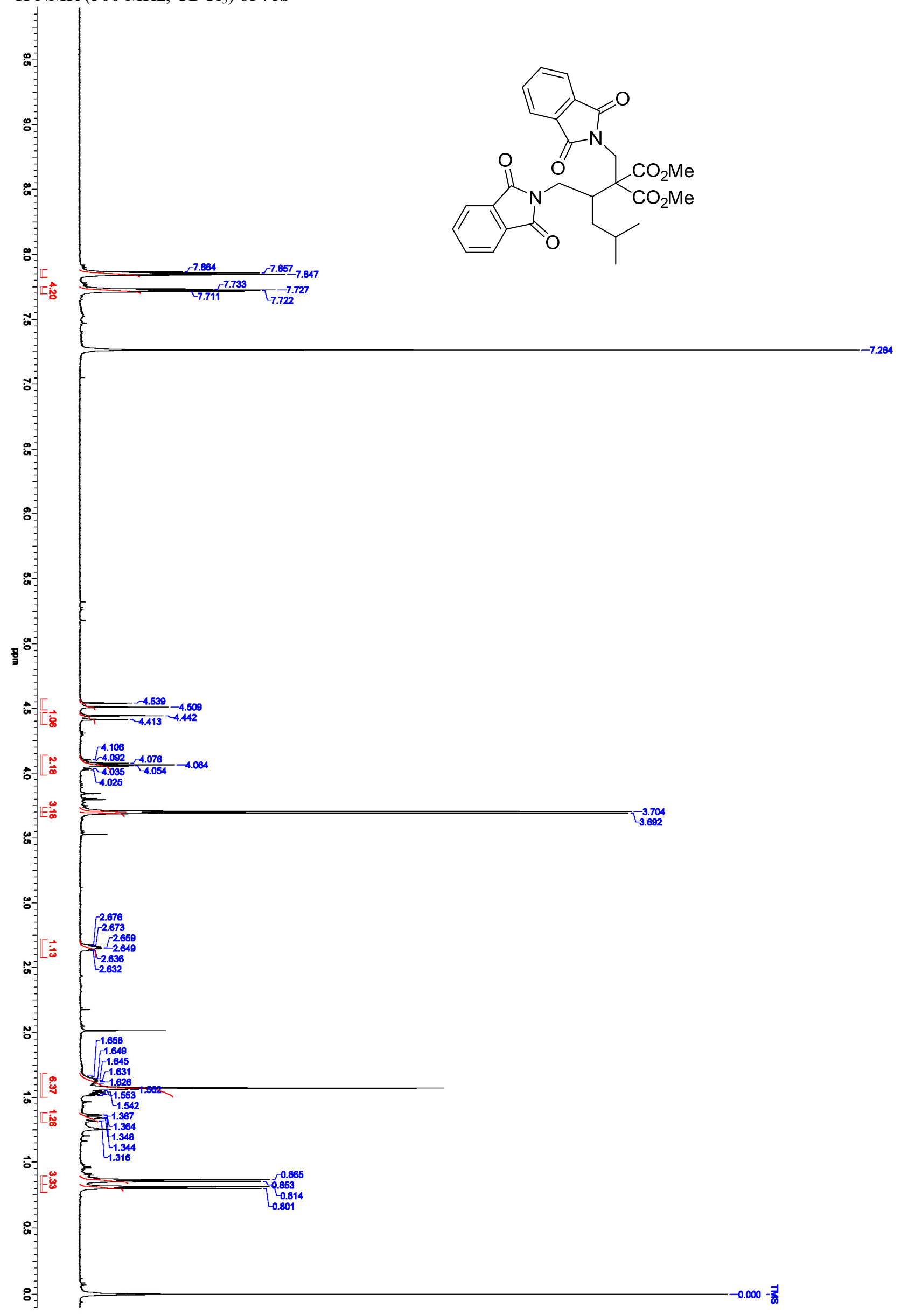


Supporting Information

${ }^{13} \mathrm{C}$ NMR (125 MHz, $\mathrm{CDCl}_{3}$ ) of 7eb

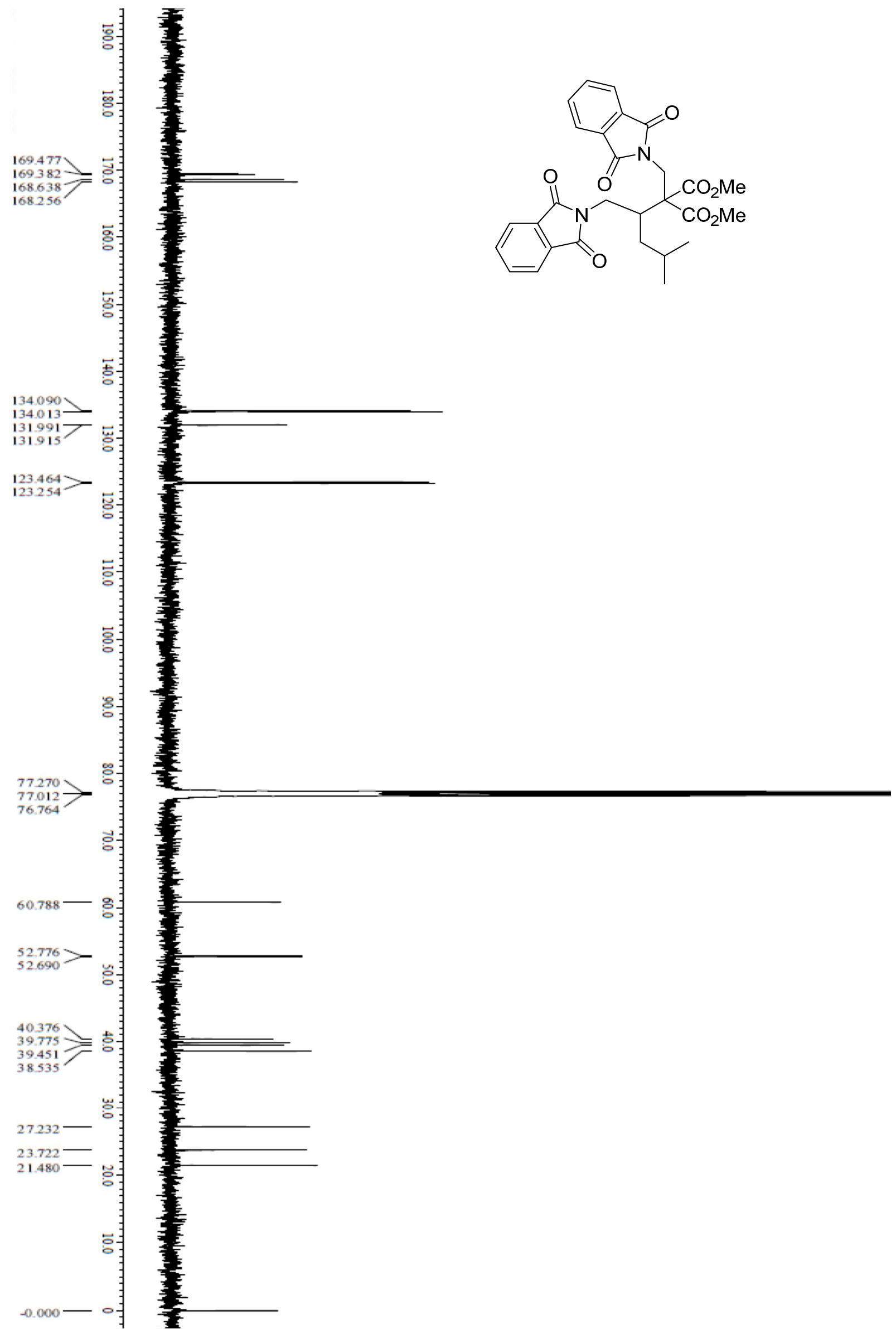


Supporting Information

${ }^{1} \mathrm{H}$ NMR $\left(500 \mathrm{MHz}, \mathrm{CDCl}_{3}\right)$ of $\mathbf{7 a c}$

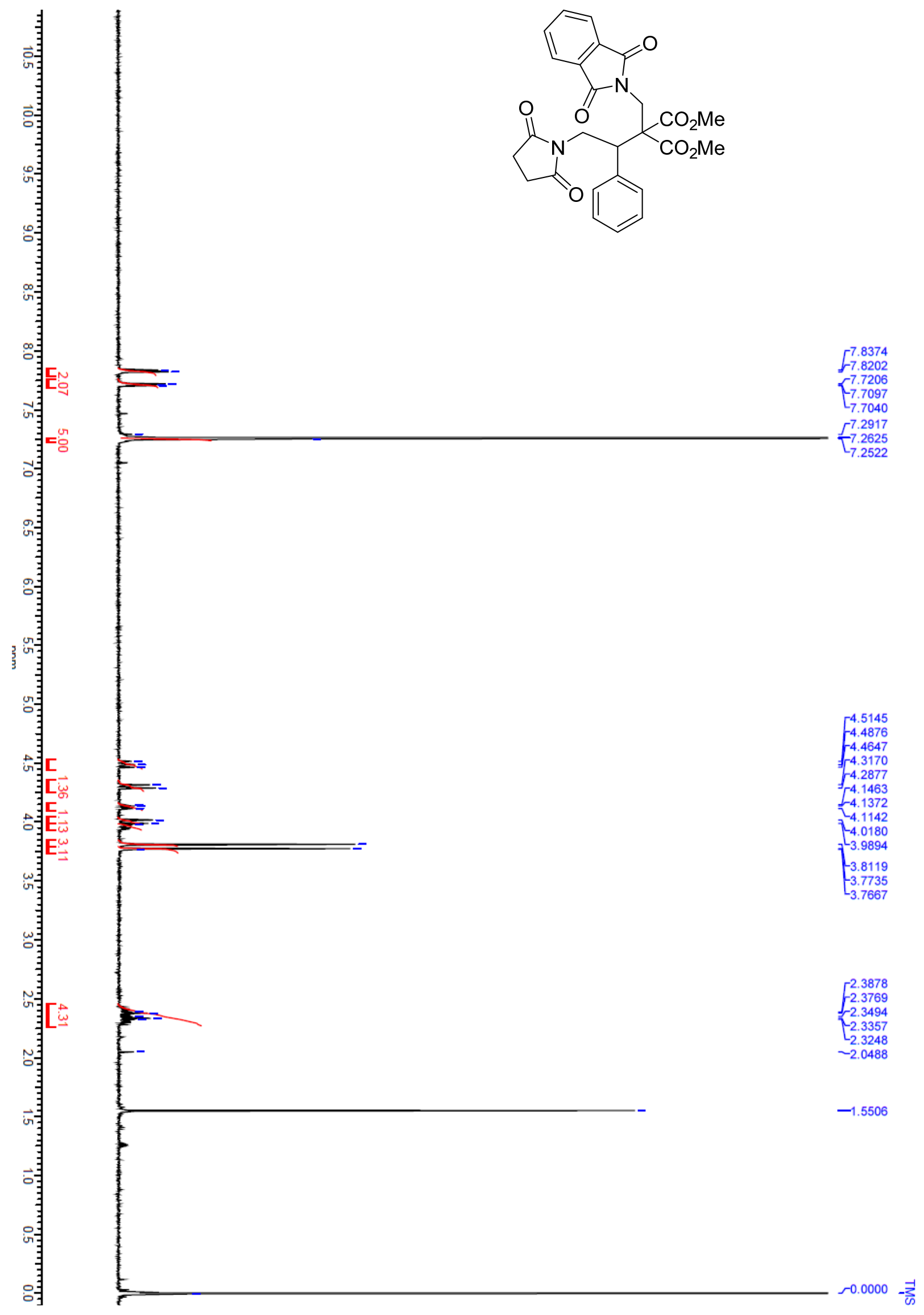


Supporting Information

${ }^{13} \mathrm{C} \mathrm{NMR}\left(125 \mathrm{MHz}, \mathrm{CDCl}_{3}\right)$ of $7 \mathbf{a c}$

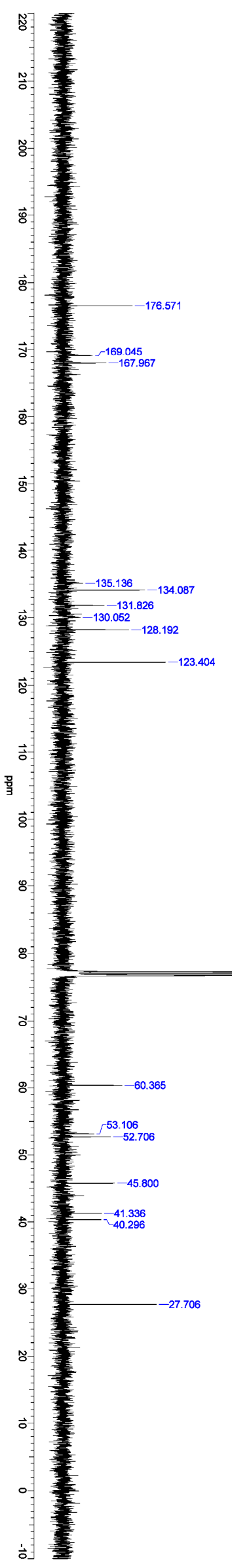<smiles>COC(=O)C(CN1C(=O)CCC1=O)(CN1C(=O)c2ccccc2C1=O)C(C)=O</smiles> 
Supporting Information

${ }^{1} \mathrm{H}$ NMR $\left(500 \mathrm{MHz}, \mathrm{CDCl}_{3}\right)$ of $\mathbf{7 a d}$

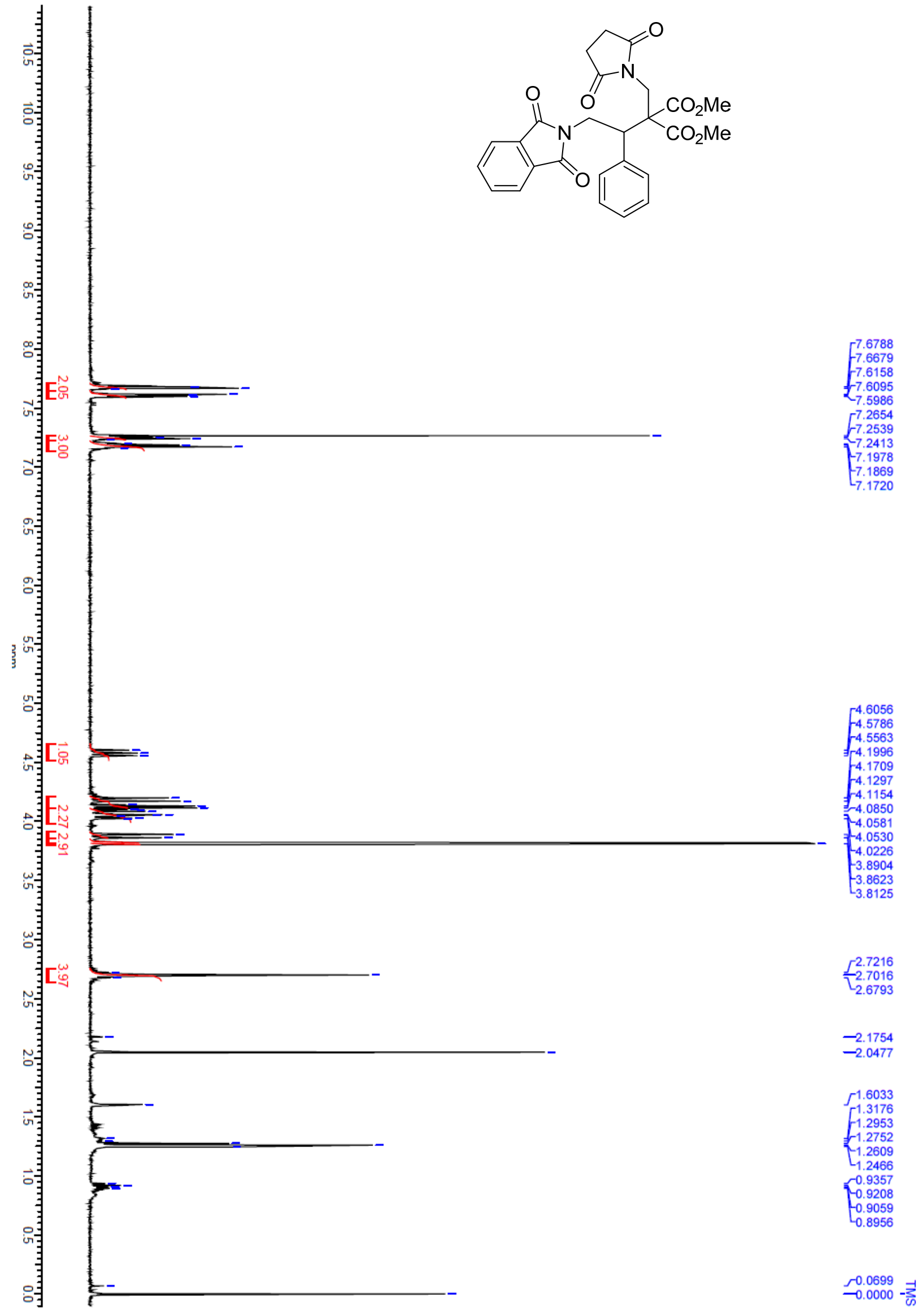




\section{Supporting Information}

${ }^{13} \mathrm{C}$ NMR $\left(500 \mathrm{MHz}, \mathrm{CDCl}_{3}\right.$ ) of $\mathbf{7 a d}$

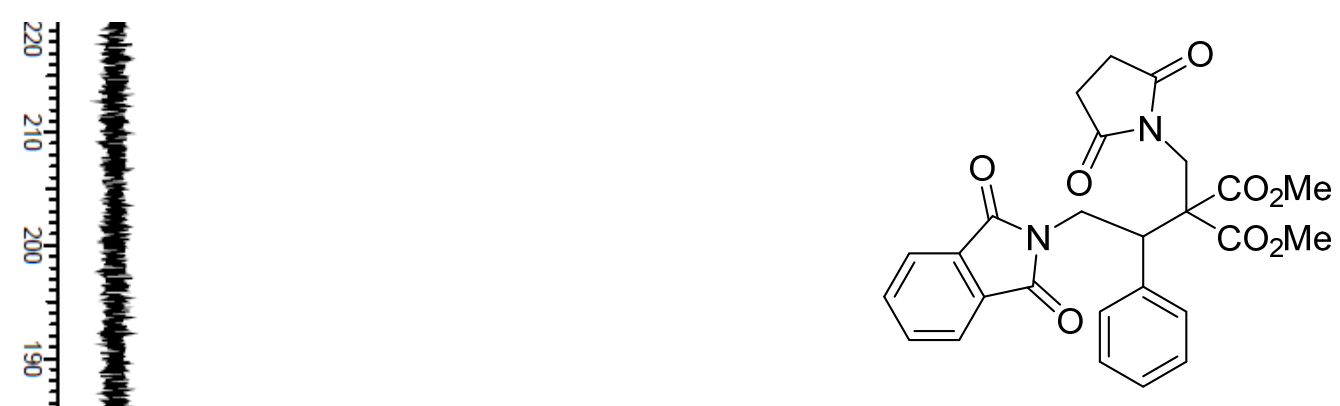

$-176.8664$

169.1880 $L_{168.9972}^{169.1880}$ ᄂ 167.7477

$\tau^{135.2506}$ $-131.6070$

$\sim 129.9473$

$-128.1541$

-128.1541
128.0587

$-123.0225$

랑

ㅎㅕㅕ

형

8

吅
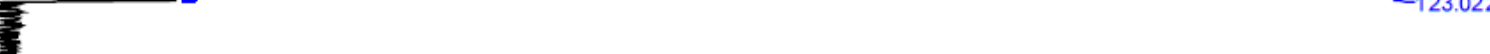

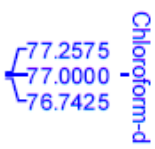

$-60.1267$

$\int^{53.1351}$

$-46.9066$

$-41.6986$

$-40.1820$

$-28.0302$ 
Supporting Information
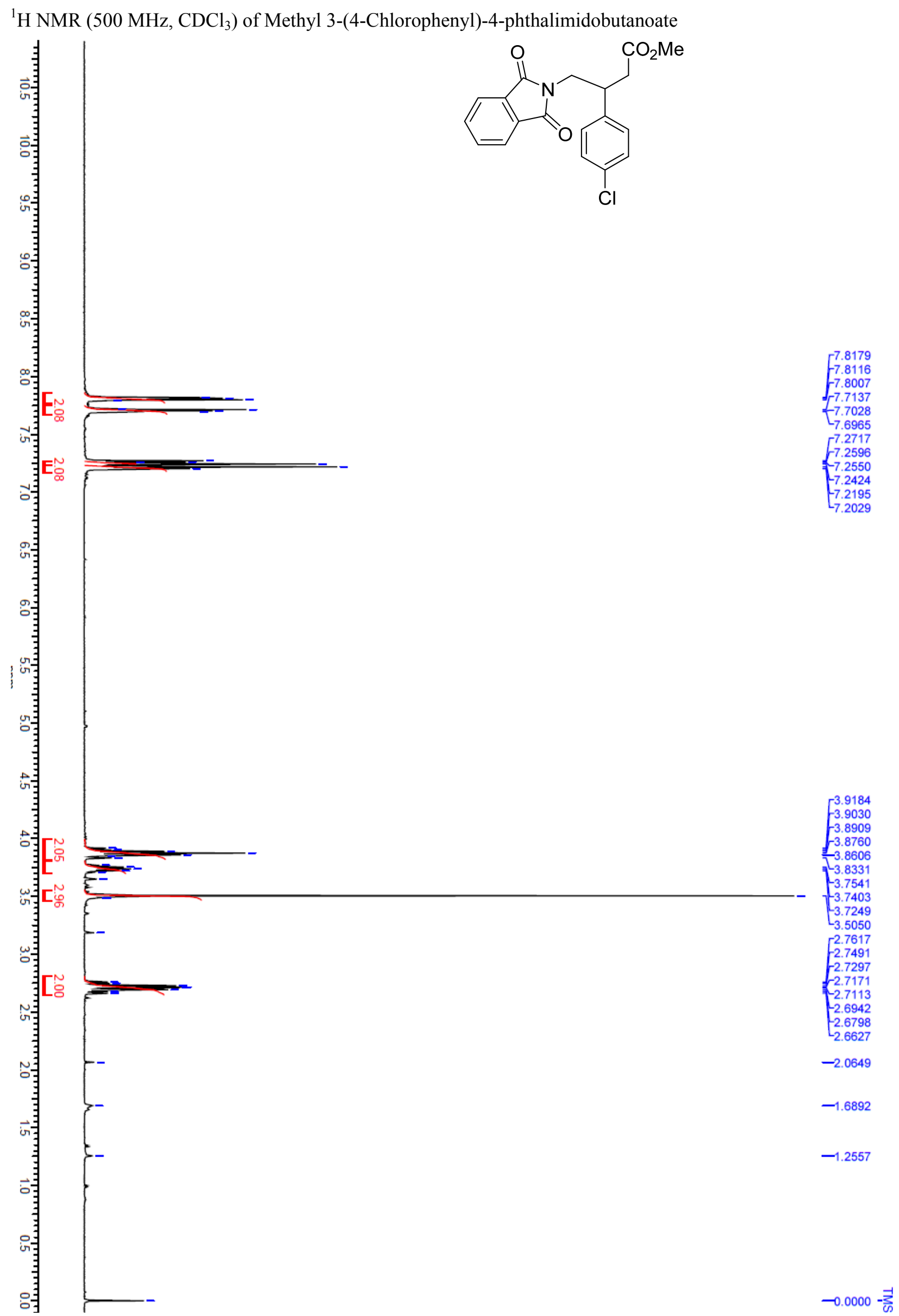

S46 


\section{Supporting Information}

${ }^{13} \mathrm{C}$ NMR (500 MHz, $\mathrm{CDCl}_{3}$ ) of Methyl 3-(4-Chlorophenyl)-4-phthalimidobutanoate

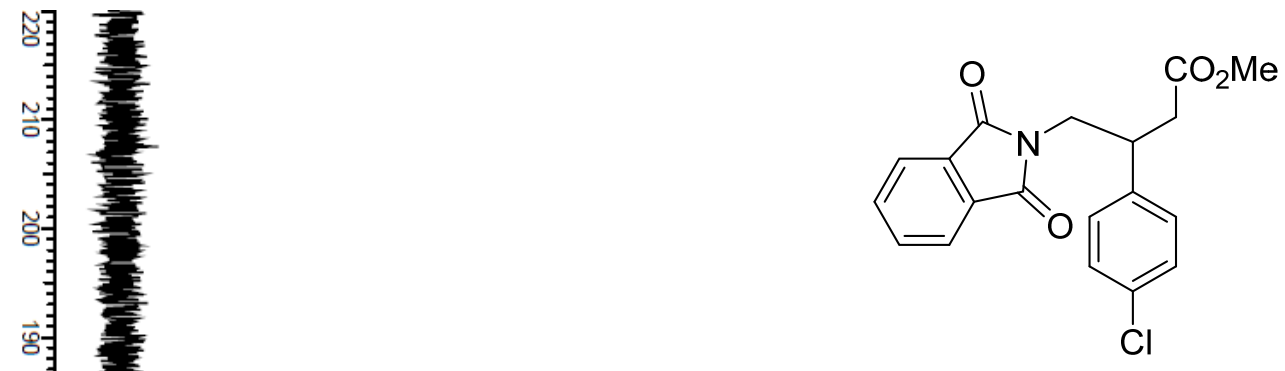

$-171.6203$

$\vec{\omega}$

愘

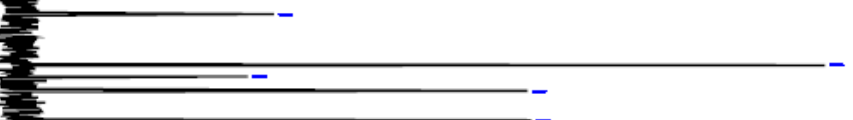

$-138.7512$

$\int_{-133.0392}^{134.0091}$

$-133.0091$

$-131.6642$

$\tau_{128.7741}^{129.0125}$

$-123.3086$

条

히

$-51.6662$ 


\section{Supporting Information}

${ }^{1} \mathrm{H}$ NMR (500 MHz, $\mathrm{CDCl}_{3}$ ) of 3-(4-Chlorophenyl)-4-phthalimidobutanoic Acid

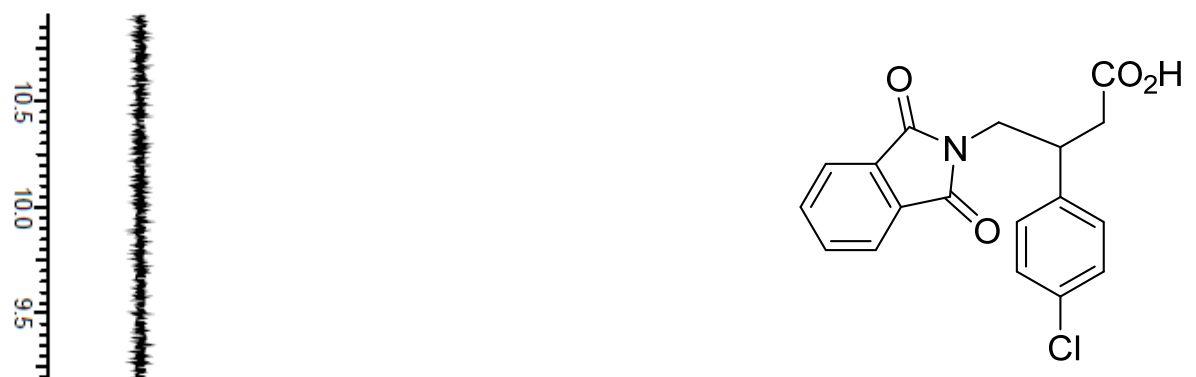




\section{Supporting Information}

${ }^{13} \mathrm{C}$ NMR (500 MHz, $\mathrm{CDCl}_{3}$ ) of 3-(4-Chlorophenyl)-4-phthalimidobutanoic Acid

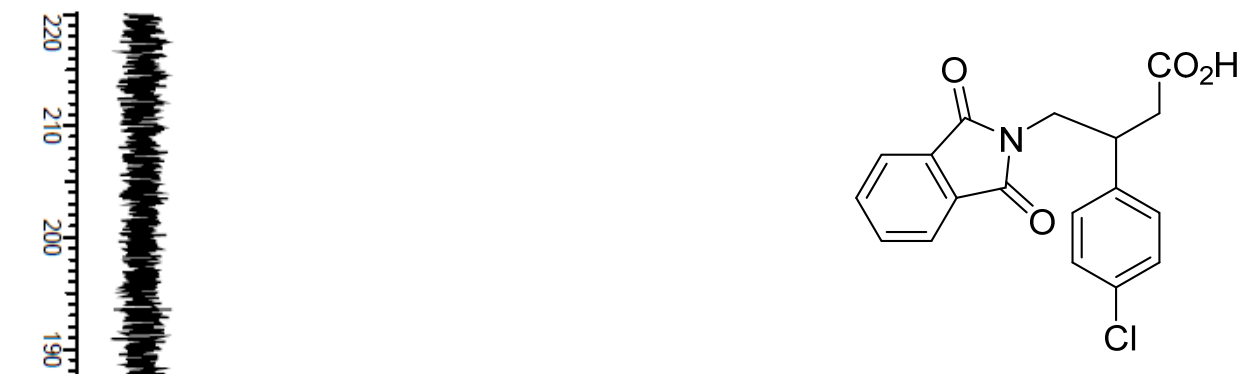


Supporting Information

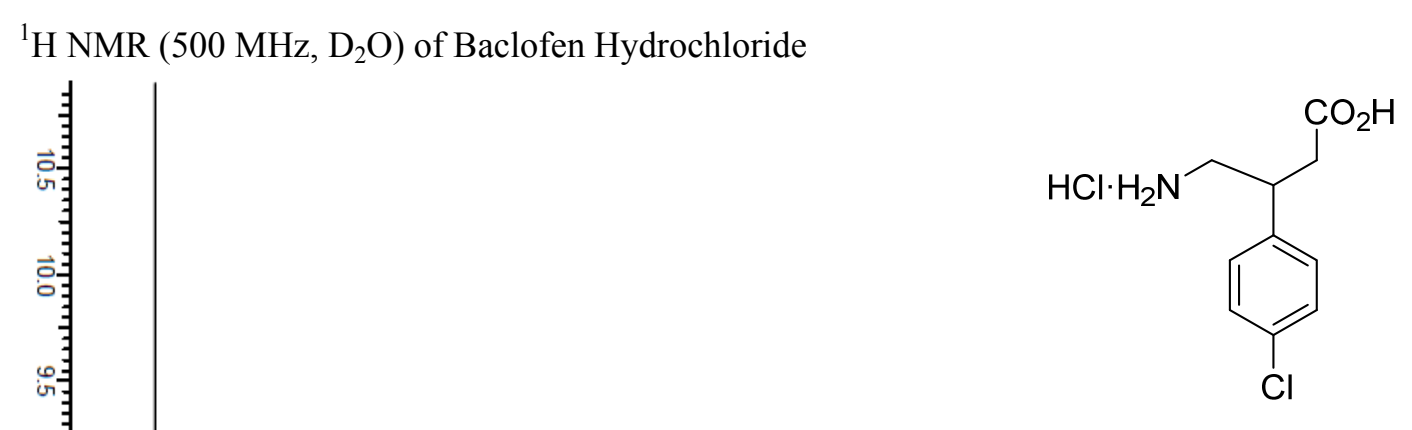

$\underline{\Gamma}_{-7.3663}^{7.3835}$

$\left[\begin{array}{r}-7.2827 \\ 7.2661\end{array}\right.$

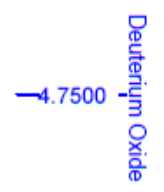

3.5006
-3.3626
-3.3311
-3.3047
-3.2652
$=-1988$
-3.1770
-2.8174
-2.7854
-2.7733
-2.7081
-2.6903
-2.6754
-2.6577
-2.6376
2.4962

S50 


\section{Supporting Information}

${ }^{1} \mathrm{H}$ NMR (500 MHz, $\mathrm{CDCl}_{3}$ ) of Methyl 5-Methyl-3-(phthalimidomethyl)hexanoate

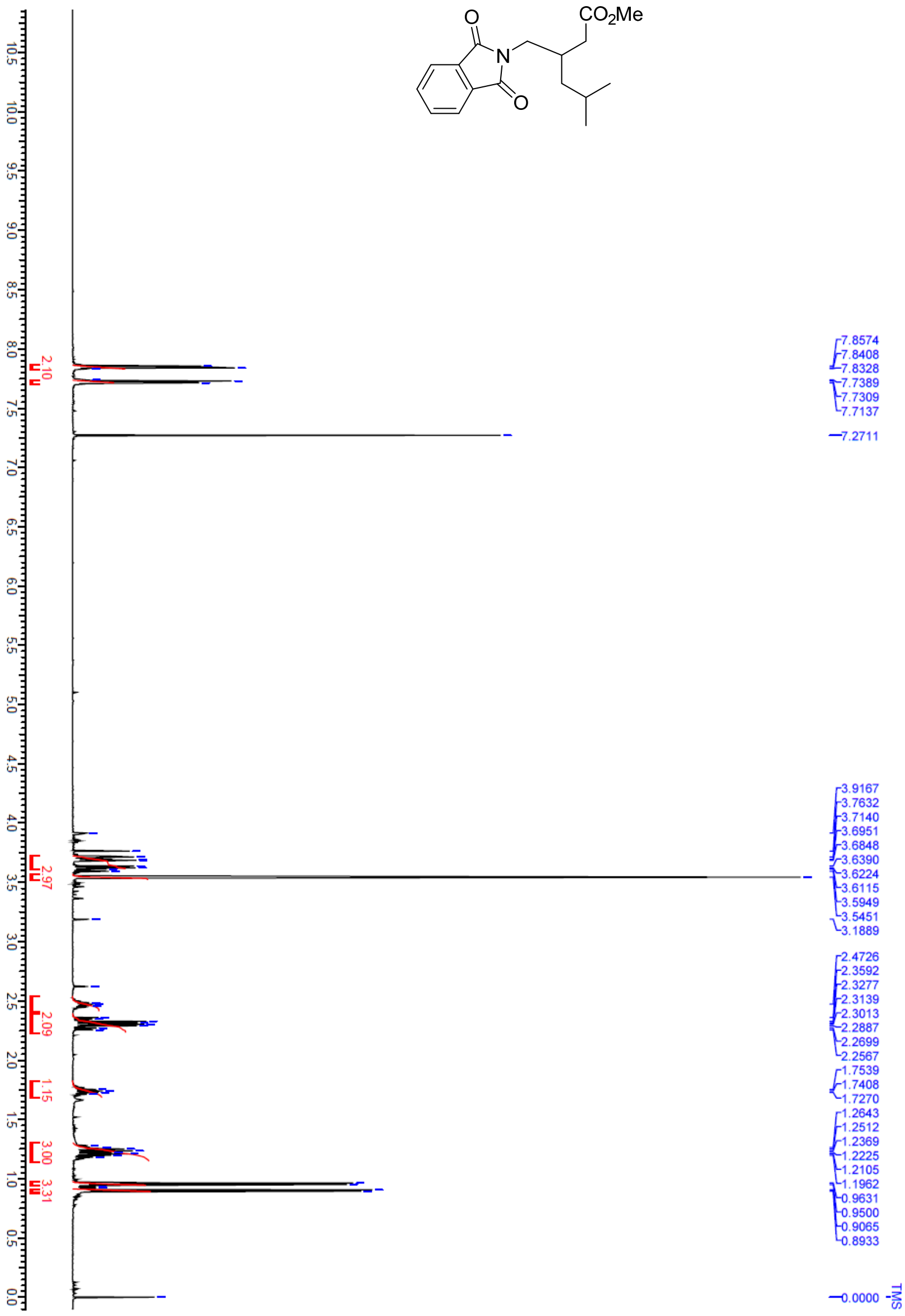




\section{Supporting Information}

${ }^{13} \mathrm{C}$ NMR (500 MHz, $\mathrm{CDCl}_{3}$ ) of Methyl 5-Methyl-3-(phthalimidomethyl)hexanoate

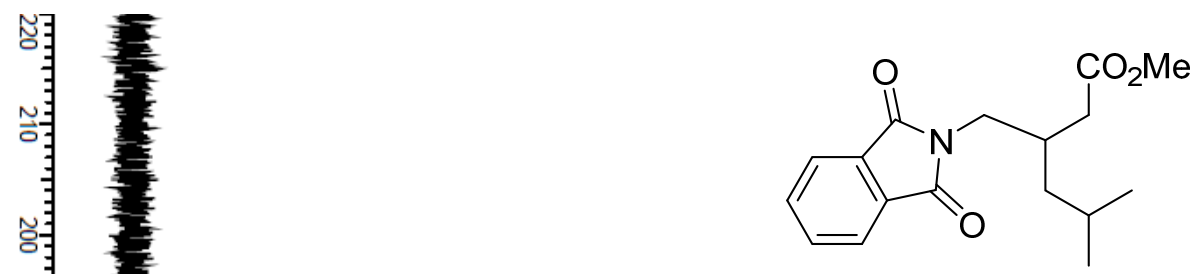




\section{Supporting Information}

${ }^{1} \mathrm{H}$ NMR (500 MHz, $\mathrm{CDCl}_{3}$ ) of 5-Methyl-3-(phthalimidomethyl)hexanoic Acid

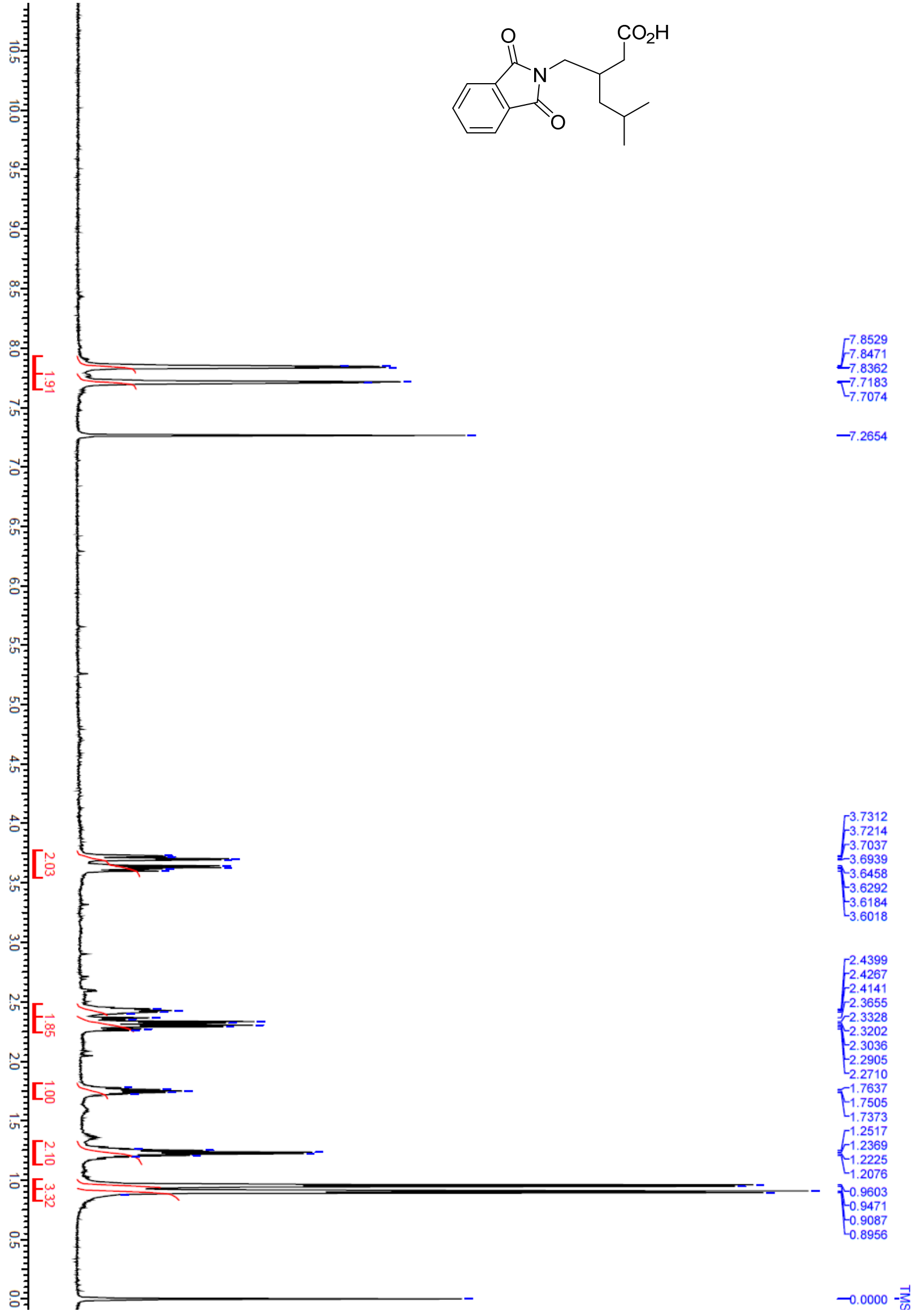




\section{Supporting Information}

${ }^{13} \mathrm{C}$ NMR (500 MHz, $\mathrm{CDCl}_{3}$ ) of 5-Methyl-3-(phthalimidomethyl)hexanoic Acid

뭉

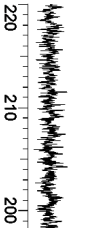

(1)

$\overrightarrow{\mathrm{\omega}}=$

$-177.343$

$\overrightarrow{\mathrm{g}}$

$-168.682$

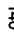

$\overrightarrow{\mathrm{\omega}}-$

$\overrightarrow{\mathrm{N}}-$

号

$\overrightarrow{\mathrm{s}}$

\&

g-

o

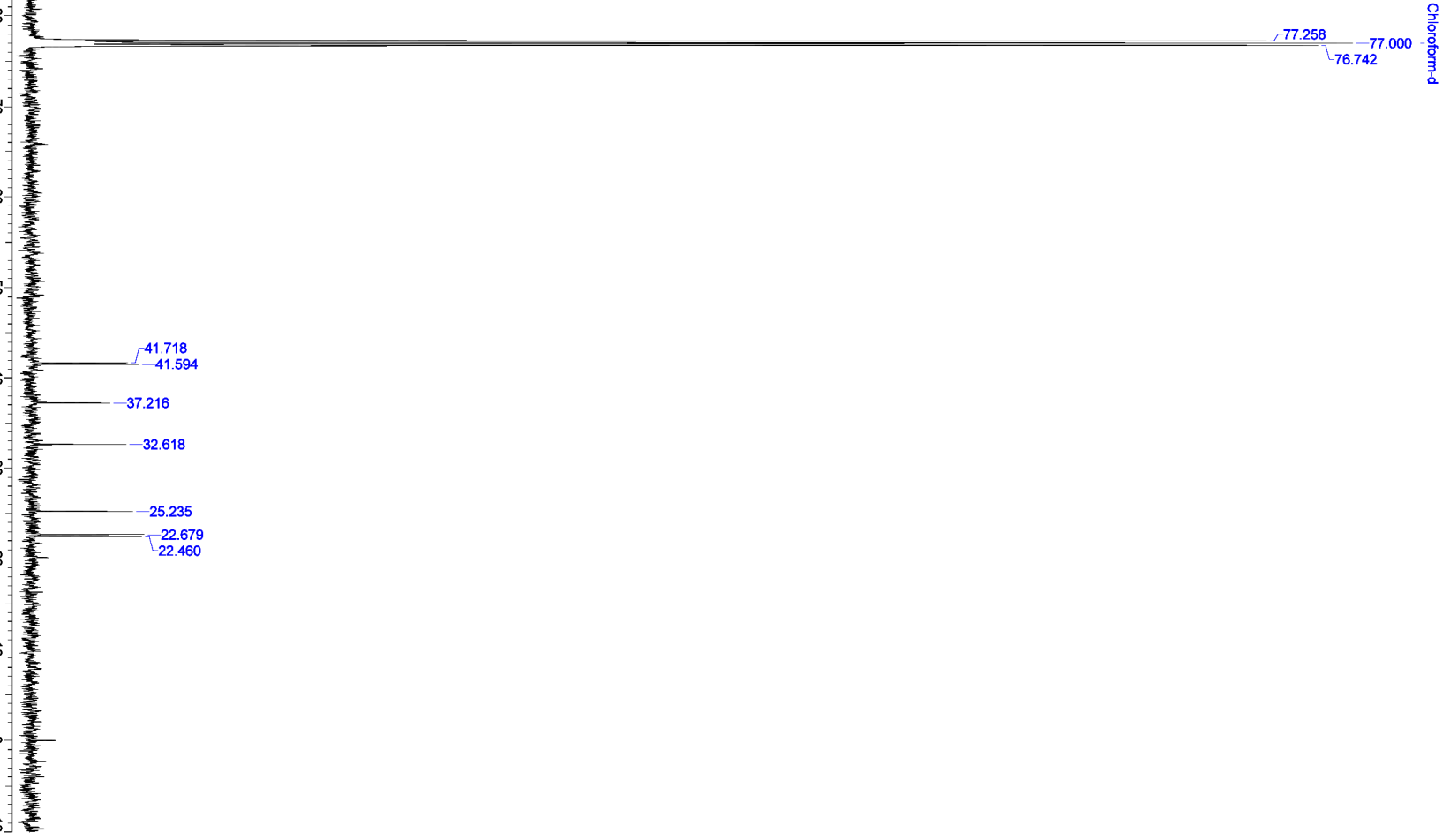


Supporting Information

${ }^{1} \mathrm{H}$ NMR (500 MHz, $\mathrm{D}_{2} \mathrm{O}$ ) of Pregabalin Hydrocholide

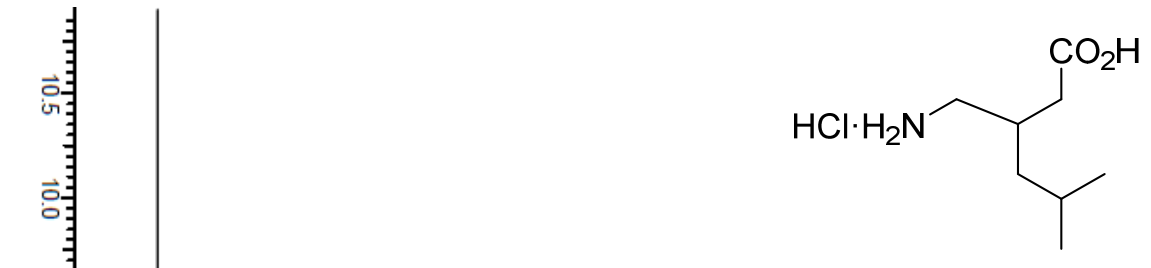


Supporting Information

${ }^{1} \mathrm{H}$ NMR $\left(500 \mathrm{MHz}, \mathrm{CDCl}_{3}\right)$ of 8

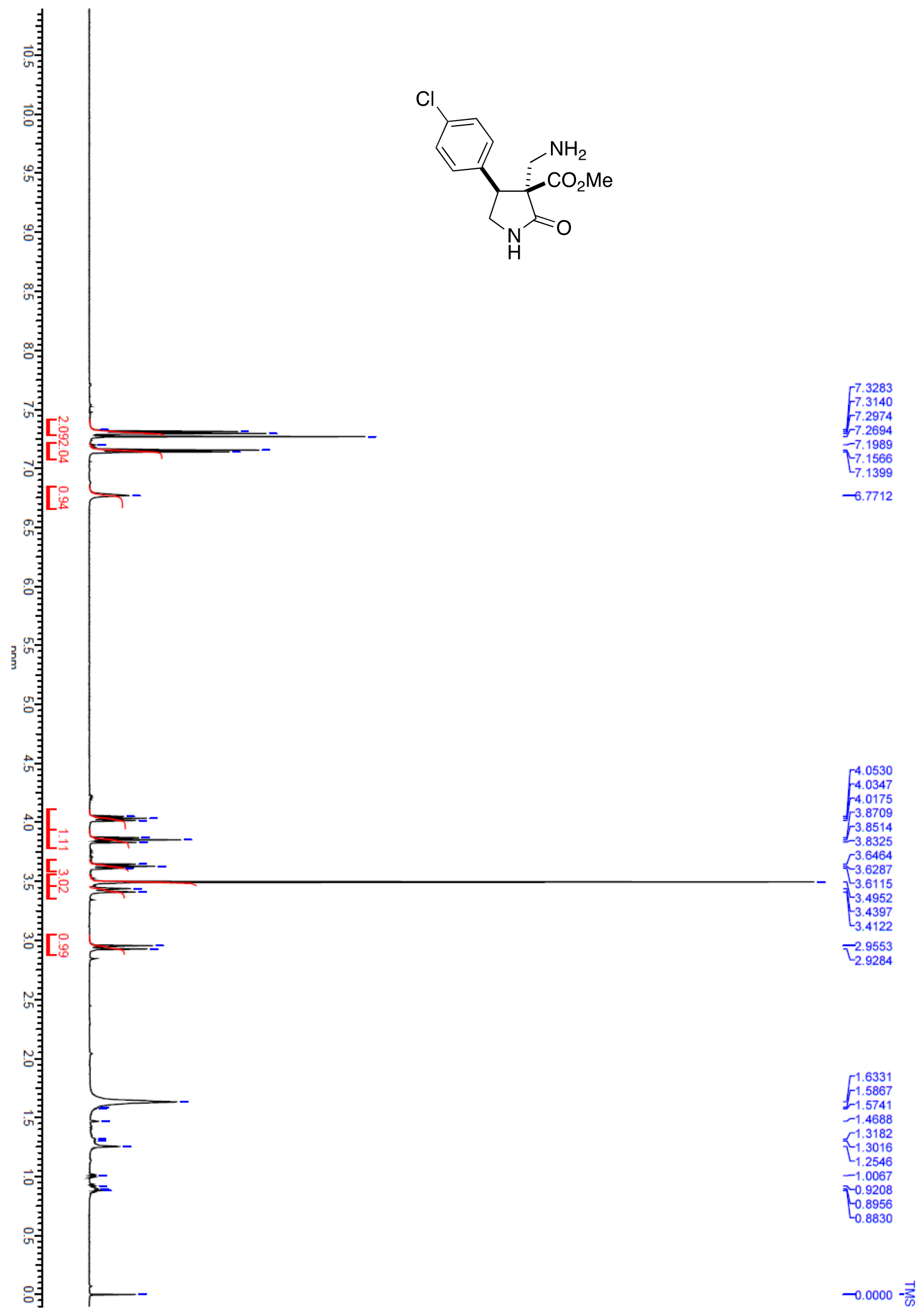


Supporting Information

${ }^{13} \mathrm{C}$ NMR (125 MHz, $\mathrm{CDCl}_{3}$ ) of 8

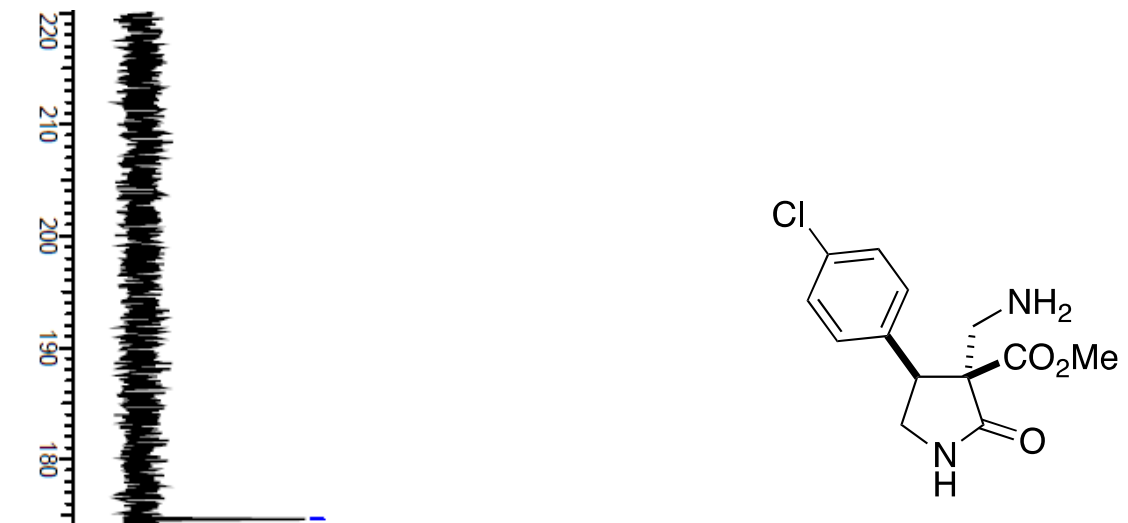

$-174.6153$

$-169.3597$

$\underline{\Gamma}^{134.7546}$

33.8294

$\Gamma_{-128.8122}^{129.4131}$

咨

혁

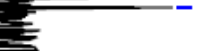

o

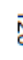

호고․

$8:$

。ำ

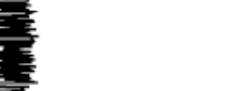

\section{o}




\section{Supporting Information}

The X-ray diffraction experiment of 8 was conducted with $\mathrm{Cu} \mathrm{K} \alpha$ radiation at $93 \mathrm{~K}$.

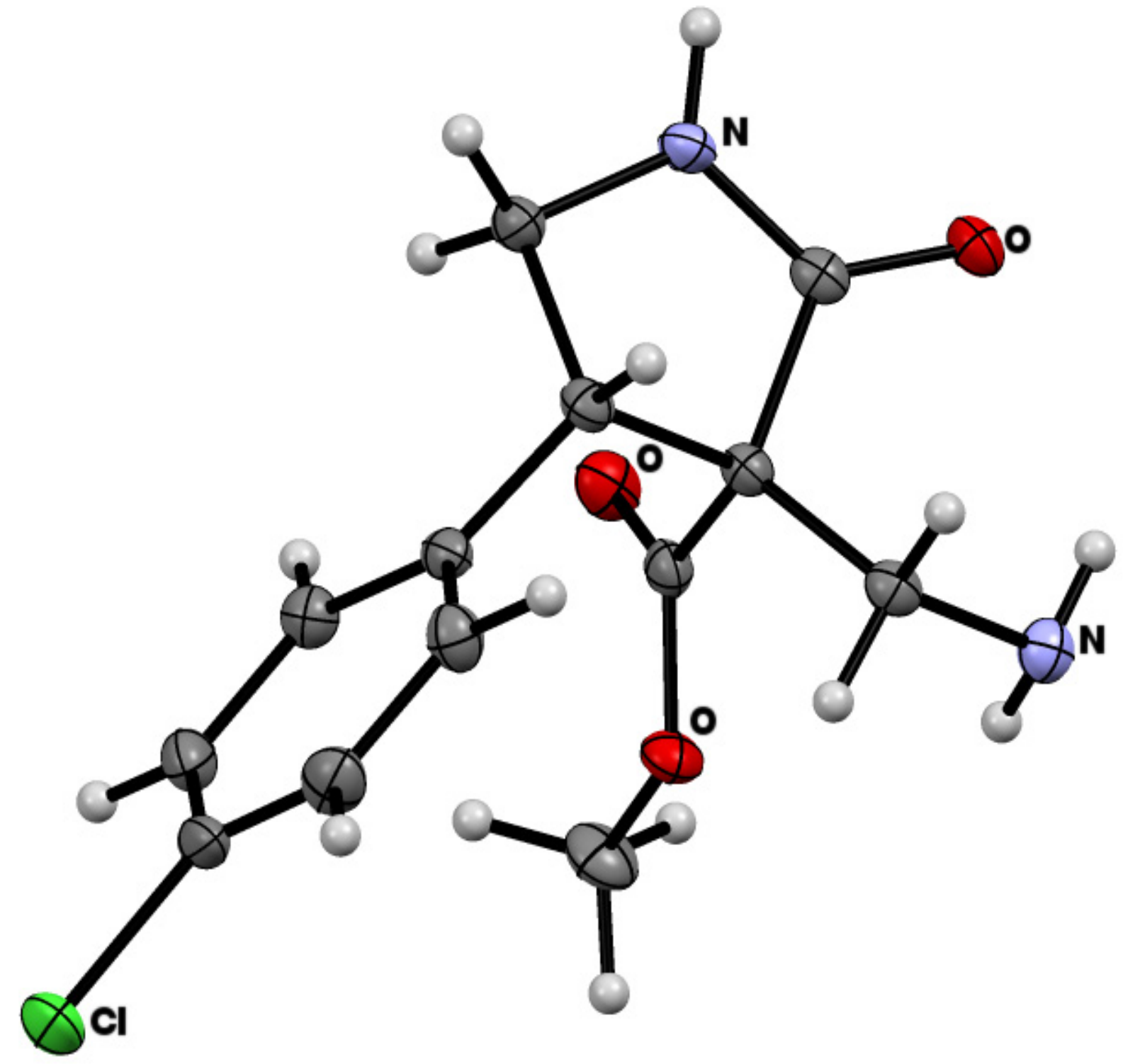

ORTEP drawing of 8 ( $50 \%$ probability).

Crystal Parameters

monoclinic, $P 2_{1} / \mathrm{c}$

$$
\begin{aligned}
& a=13.361(8), b=11.884(9), c=8.306(7) \\
& \alpha=90.0, \beta=90.210(15), \gamma=90.0 \\
& V=1318.8(17) \AA^{3}, Z=4, D x=1.424 \mathrm{~g} / \mathrm{cm}^{3}
\end{aligned}
$$

Refinement Metrics

Data Completeness $=0.993 R=0.0345, \mathrm{w} R 2=0.0936, S=1.111$

See the separate CIF file for further details. 


\section{Supporting Information}

\section{Reference}

(1) Frisch, M. J.; Trucks, G. W.; Schlegel, H. B.; Scuseria, G. E.; Robb, M. A.; Cheeseman, J. R.; Montgomery, Jr., J. A.; Vreven, T.; Kudin, K. N.; Burant, J. C.; Millam, J. M.; Iyengar, S. S.; Tomasi, J.; Barone, V.; Mennucci, B.; Cossi, M.; Scalmani, G.; Rega, N.; Petersson, G. A.; Nakatsuji, H.; Hada, M.; Ehara, M.; Toyota, K.; Fukuda, R.; Hasegawa, J.; Ishida, M.; Nakajima, T.; Honda, Y.; Kitao, O.; Nakai, H.; Klene, M.; Li, X.; Knox, J. E.; Hratchian, H. P.; Cross, J. B.; Bakken, V.; Adamo, C.; Jaramillo, J.; Gomperts, R.; Stratmann, R. E.; Yazyev, O.; Austin, A. J.; Cammi, R.; Pomelli, C.; Ochterski, J. W.; Ayala, P. Y.; Morokuma, K.; Voth, G. A.; Salvador, P.; Dannenberg, J. J.; Zakrzewski, V. G.; Dapprich, S.; Daniels, A. D.; Strain, M. C.; Farkas, O.; Malick, D. K.; Rabuck, A. D.; Raghavachari, K.; Foresman, J. B.; Ortiz, J. V.; Cui, Q.; Baboul, A. G.; Clifford, S.; Cioslowski, J.; Stefanov, B. B.; Liu, G.; Liashenko, A.; Piskorz, P.; Komaromi, I.; Martin, R. L.; Fox, D. J.; Keith, T.; Al-Laham, M. A.; Peng, C. Y.; Nanayakkara, A.; Challacombe, M.; Gill, P. M. W.; Johnson, B.; Chen, W.; Wong, M. W.; Gonzalez, C.; Pople, J. A.; Gaussian, Inc., Wallingford CT, 2004. 